\title{
Using a Temporary Silicon Connection in Stereoselective Allylation with Allylsilanes: Application to the Synthesis of Stereodefined 1,2,4-Triols
}

\author{
Julien Beignet, Liam R. Cox* \\ School of Chemistry, University of Birmingham, Edgbaston, Birmingham, B15 2TT, UK.
}

Email: I.r.cox@bham.ac.uk

\section{Supporting information:}

General experimental procedures for the allylation of aldehydes 6, Tamao oxidation of the cyclization products 7 and acetonide formation from triols 9 .

Characterization data for allylation products, triols and acetonide derivatives $\left({ }^{1} \mathrm{H}-\mathrm{NMR}\right.$ and ${ }^{13} \mathrm{C}$ NMR only)

\section{General Information}

All characterizations were carried out at the School of Chemistry, University of Birmingham, UK.

Elemental analyses were recorded on a Carlo Erba EA1110 simultaneous CHNS analyser. Infrared spectra were recorded neat as thin films between sodium chloride discs on a Perkin Elmer 1600 FTIR spectrometer. The intensity of each band is described as $\mathrm{s}$ (strong), $\mathrm{m}$ (medium) or w (weak) and with the prefix $v$ (very) and suffix br (broad) where appropriate. ${ }^{1} \mathrm{H}-\mathrm{NMR}$ and ${ }^{13} \mathrm{C}-\mathrm{NMR}$ spectra were recorded at 500 and $125 \mathrm{MHz}, 400$ and $100 \mathrm{MHz}$ or 300 and $75 \mathrm{MHz}$, respectively, using Bruker DRX 500, Bruker AMX 400, Bruker AV 400, Bruker AV 300 and Bruker AC 300 spectrometers. Chemical shifts are reported as $\delta$ values (ppm) referenced to the following solvent signals: $\mathrm{CHCl}_{3}, \delta 7.26 ; \mathrm{CDCl}_{3}$, $\delta$ 77.0. Acetonides 11 and 12 were recorded in $\mathrm{d}_{6}$-benzene: $\mathrm{C}_{6} \mathrm{H}_{6}, \delta 7.16 ; \mathrm{C}_{6} \mathrm{D}_{6}, \delta$ 128.0. The term, 'stack' is used to describe a region where resonances arising from non-equivalent nuclei are coincident, and multiplet, $\mathrm{m}$, to describe a region where resonances arising from a single nucleus (or equivalent nuclei) are coincident but coupling constants cannot be readily assigned. Connectivities were deduced from COSY90, HSQC and HMBC experiments. GOESY and NOESY experiments were used to elucidate relative stereochemistry. Mass spectra were recorded on a Micromass LCT spectrometer utilizing electrospray ionization (and a methanol mobile phase), and Micromass Prospec and Zabspec spectrometers utilizing electron impact and/or chemical ionization (ammonia as the carrier gas). HRMS were recorded on a Micromass LCT spectrometer using a lock mass incorporated into the mobile phase. 
Preparative HPLC was performed on a reverse phase Phenomenex Luna 10u C18(2)100A $50 \times 21$, $20 \mathrm{~mm} \times 10$ micron fitted with a Dionex P580 pump and a Dionex UVD170S detector (used at 210 and $225 \mathrm{~nm}$ ) using a helium-degassed HPLC grade water/acetonitrile gradient, without acidic additives. Elution was monitored and spectra were recorded on Dionex Chromeleon 6.11 software.

\section{Reactions}

All reactions were conducted in oven-dried $\left(140^{\circ} \mathrm{C}\right)$ or flame-dried Schlenk glassware under a nitrogen atmosphere, and at ambient temperature $\left(20\right.$ to $\left.25^{\circ} \mathrm{C}\right)$ unless otherwise stated, with magnetic stirring. Volumes of $5 \mathrm{~cm}^{3}$ or less were measured and dispensed with Hamilton gastight syringes. Reactions were monitored by thin layer chromatography using pre-coated glass-backed ICN silicarapid plates $\left(60 \mathrm{~A} \mathrm{~F}_{254}\right)$ and visualized by UV detection $(254 \mathrm{~nm})$ and potassium manganate $(\mathrm{VII})$ and/or ammonium molybdate(IV) - cerium(IV) sulfate dips. Column chromatography was performed on Merck silica gel (particle size 40-63 $\mu \mathrm{m}$ mesh) or Fluka 60 (40-60 $\mu \mathrm{m}$ mesh) silica gel. Evaporation and concentration under reduced pressure was performed at 50 - 500 mbar. Residual solvent was removed under high vacuum (1 mbar).

\section{Materials}

All reagents were obtained from commercial sources and used without further purification unless stated otherwise. 2,4,6-Tri-tert-butylprimidine (TTBP) was synthesized following a known procedure. ${ }^{1}$ Trimethylsilyl chloride was distilled under nitrogen from $\mathrm{CaH}_{2}$ and stored under nitrogen at $4{ }^{\circ} \mathrm{C}$. Trimethylsilyl trifluoromethanesulfonate (triflate) was stored in a Schlenk tube under nitrogen at $4{ }^{\circ} \mathrm{C}$ and used whilstever the compound was colorless. Over time a pink coloration was observed whereupon fresh TMSOTf was used. Diethylamine and $N, N^{\prime}, N^{\prime}, N^{\prime}-$ tetramethylethylenediamine were distilled under a nitrogen atmosphere from $\mathrm{KOH}$ and stored under nitrogen at room temperature over activated $4 \AA$ molecular sieves (activated by heating under a vacuum for $15 \mathrm{~min}$ with a bunsen flame immediately before use). Dichloromethane and acetonitrile were freshly distilled under nitrogen from $\mathrm{CaH}_{2}$. Tetrahydrofuran and diethyl ether were freshly distilled under nitrogen from sodium benzophenone ketyl. All solutions are aqueous and saturated unless stated otherwise.

\section{General procedure for allylation reaction: synthesis of oxasilacycles $7 \mathrm{a}-\mathrm{i}$ and $\mathbf{8 a}-\mathbf{i}$.}

TMSOTf (1.0 equiv.) was added dropwise (approximately one drop per second) via syringe to a solution of aldehyde 6a-i (1.0 equiv.) and 2,6-di-tert-butyl-4-methylpyridine (2,6-DTBMP) (1.2 equiv.) in $\mathrm{CH}_{2} \mathrm{Cl}_{2}\left(0.1 \mathrm{M}\right.$ reaction concentration) at $-78{ }^{\circ} \mathrm{C}$. The reaction mixture was stirred at $-78{ }^{\circ} \mathrm{C}$. TLC indicated consumption of starting material within 8-16 $\mathrm{h}$. The reaction was then quenched by adding

\footnotetext{
${ }^{1}$ Crich, D.; Smith, M.; Yao, Q.; Picione, J, Synthesis 2001, 323-326.
} 
an equivalent volume of $\mathrm{NaHCO}_{3}$ solution at $-78^{\circ} \mathrm{C}$ and allowed to warm to room temperature over 30 min. The two phases were separated and the aqueous phase extracted with $\mathrm{CH}_{2} \mathrm{Cl}_{2}(2 \times$ volume of aqueous phase). The combined organic extracts were successively washed with water (1/3 volume of organic phase) and brine (1/3 volume of organic phase) and dried over $\mathrm{MgSO}_{4}$. Filtration and evaporation of the volatiles under reduced pressure on a rotary evaporator provided a yellow oil (quantitative mass recovery). This was used in the following Tamao oxidation without further purification. Analytically pure samples of each oxasilacycle were obtained by preparative HPLC. This allylation reaction was performed on scales ranging from $0.15 \mathrm{mmol}$ to $15 \mathrm{mmol}$ of aldehyde without any noticeable changes in rates, yields or ratios of products. All reactions were carried out a minimum of two times.

\section{General procedure for Tamao oxidation: formation of triols 9 .}

$\mathrm{H}_{2} \mathrm{O}_{2}$ (20 equiv., $60 \%$ in $\mathrm{H}_{2} \mathrm{O}$ ), $\mathrm{KHCO}_{3}$ (3.0 equiv.) and $\mathrm{KF}$ (5.0 equiv.) were added to a solution of the products from the allylation of aldehyde 6a-i (1 equiv.) in MeOH:THF (1:1) (0.1 M reaction concentration) and the resulting mixture was stirred at room temperature in a round-bottom flask. The reaction was monitored by TLC and consumption of the starting material occurred within 4 to 7 days (an additional 5 equiv. of $\mathrm{H}_{2} \mathrm{O}_{2}$ were sometimes added after a few days to drive the reaction to completion). The mixture was then poured into an equal volume of $\mathrm{Na}_{2} \mathrm{~S}_{2} \mathrm{O}_{3}$ solution and stirred for 30 min. The resulting mixture was extracted with EtOAc $(3 \times 2$ volumes $)$ and the combined organic extracts were washed with brine ( 1/6 volume of EtOAc) and dried over $\mathrm{MgSO}_{4}$. Filtration and evaporation of the volatiles under reduced pressure on a rotary evaporator afforded the triol $\mathbf{9}$ as a yellow oil which was further purified by flash column chromatography. A short column and gradient elution were used to minimize losses of the trio/ $9 \mathrm{a}-\mathrm{h}$ on the silica.

\section{General procedure for Acetonide formation: formation of acetonides 11 and 12.}

$\mathrm{Na}_{2} \mathrm{SO}_{4}(40 \mathrm{mg})$ and $p \mathrm{TsOH} \cdot \mathrm{H}_{2} \mathrm{O}(2 \mathrm{mg}, 16 \mu \mathrm{mol})$ were added to a solution of trio/ $9 \mathrm{a}-\mathrm{h}(30 \mathrm{mg})$ in acetone (non redistilled, 1.0-2.0 mL) and the resulting mixture was stirred overnight. The reaction mixture was poured over a $\mathrm{NaHCO}_{3}$ solution $(3 \mathrm{~mL})$ and the layer was extracted with EtOAc $(3 \times 10$ $\mathrm{mL})$. The combined organic extracts were washed with brine $(5 \mathrm{~mL})$ and dried over $\mathrm{MgSO}_{4}$. Filtration and evaporation of the volatiles under reduced pressure on a rotary evaporator left a mixture of acetonides 11 and 12 as a pale yellow oil in quantitative yield. The mixture was then used without further purification for NMR spectroscopic studies (in $\mathrm{C}_{6} \mathrm{D}_{6}$ ).

\section{Allylation reaction of Aldehyde 6a:}

TMSOTf $(2.37 \mathrm{~mL}, 13.1 \mathrm{mmol})$ was added to a solution of aldehyde $6 \mathrm{a}(4.60 \mathrm{~g}, 13.1 \mathrm{mmol})$ and TTBP (2.96 g, $14.4 \mathrm{mmol})$ in $\mathrm{CH}_{2} \mathrm{Cl}_{2}(131 \mathrm{~mL})$ at $-78{ }^{\circ} \mathrm{C}$ and the reaction mixture was stirred for $8 \mathrm{~h}$. Aqueous work-up and removal of the solvent afforded compounds $7 \mathbf{a}$ and $8 \mathbf{a}$ (70:18; inseparable mixture) as a colorless liquid $(7.51 \mathrm{~g}, 88 \%$, resulting mass being diene products). This was used in the 
following step without any further purification. Analytically pure samples of each compound could not be obtained by preparative HPLC ( $\mathrm{t}=0 \rightarrow 50 \mathrm{~min}, 0 \rightarrow 100 \% \mathrm{MeCN}$ in $\left.\mathrm{H}_{2} \mathrm{O}\right) ; 7 \mathrm{a} t_{\mathrm{R}}=68.3 \mathrm{~min}$ (contaminated with diene $\mathbf{5 a}$ ) and $\mathbf{8 a} t_{\mathrm{R}}=70.4 \mathrm{~min}$ (contaminated with residual TTBP).

\section{$\left(3 S^{*}, 4 R^{*}, 6 S^{\star}\right)$ oxasilinane $7 a$}<smiles>C=CC1[C@H](OC)CC(c2ccccc2)O[Si]1(CC)CC</smiles>

Exact Mass: 348.19408

Mol. Wt.: 348.62718

C, 65.46; H, 9.25; O, 9.18; Si, 16.11\%

HPLC: $t_{\mathrm{R}}=68.3 \mathrm{~min} ; v_{\max }(\mathrm{film}) / \mathrm{cm}^{-1} 3075 \mathrm{w}, 3029 \mathrm{w}, 2956 \mathrm{~s}, 2916 \mathrm{~m}, 2877 \mathrm{~s}, 1627 \mathrm{~m}(\mathrm{C}=\mathrm{C}), 1494 \mathrm{w}$, $1453 \mathrm{~m}, 1415 \mathrm{~m}, 1364 \mathrm{w}, 1328 \mathrm{w}, 1309 \mathrm{w}, 1252 \mathrm{~s}, 1200 \mathrm{w}, 1146 \mathrm{~m}, 1003 \mathrm{~s}, 939 \mathrm{~m}, 887 \mathrm{~m}, 871 \mathrm{~m}, 842 \mathrm{~s}$, $800 \mathrm{~m}, 745 \mathrm{~m}, 698 \mathrm{~s} ; \delta_{\mathrm{H}}(300 \mathrm{MHz}) 0.14\left(9 \mathrm{H}, \mathrm{s}, \mathrm{Si}\left(\mathrm{CH}_{3}\right)_{3}\right), 0.62-0.75\left(2 \mathrm{H}, \mathrm{m}, \mathrm{OSi}\left(\mathrm{CH}_{2} \mathrm{CH}_{3}\right)\right), 0.80-0.93$ $\left(2 \mathrm{H}, \mathrm{m}, \mathrm{OSi}\left(\mathrm{CH}_{2} \mathrm{CH}_{3}\right)\right), 1.01\left(3 \mathrm{H}, \mathrm{t}, J\right.$ 8.1, OSi $\left.\left(\mathrm{CH}_{2} \mathrm{CH}_{3}\right)\right), 1.04\left(3 \mathrm{H}, \mathrm{t}, J\right.$ 8.1, OSi $\left.\left(\mathrm{CH}_{2} \mathrm{CH}_{3}\right)\right), 1.82(1 \mathrm{H}$, ddd, J 14.1, 5.4, 2.7, 5- $\left.H_{\text {pseudoeq. }}\right), 1.91-2.03\left(2 \mathrm{H}\right.$, stack, 3-H, 5- $\left.H_{\text {pseudoax. }}\right), 4.13$ (1H, app. t, J 4.4, 4-H), $4.96\left(1 \mathrm{H}, \mathrm{d}, J 10.5,1-H_{\text {cis }}\right), 4.98\left(1 \mathrm{H}, \mathrm{d}, J\right.$ 16.2, 1- $\left.H_{\text {trans }}\right), 5.29(1 \mathrm{H}, \mathrm{dd}, J$ 9.6, 2.7, 6-H), $5.87(1 \mathrm{H}$, app. $\mathrm{dt}, J$ 16.2, 10.5, 2-H), 7.12-7.42 $(5 \mathrm{H}$, stack, $\mathrm{PhH}) ; \delta_{\mathrm{C}}(75 \mathrm{MHz}) 0.1\left(\mathrm{CH}_{3}, \mathrm{Si}\left(\mathrm{CH}_{3}\right)_{3}\right), 5.0\left(2 \times \mathrm{CH}_{2}\right.$, OSi $\left.\left(\mathrm{CH}_{2} \mathrm{CH}_{3}\right)_{2}\right), 6.7\left(\mathrm{CH}_{3}, \mathrm{OSi}\left(\mathrm{CH}_{2} \mathrm{CH}_{3}\right)\right), 6.9\left(\mathrm{CH}_{3}, \mathrm{OSi}\left(\mathrm{CH}_{2} \mathrm{CH}_{3}\right)\right), 38.5(\mathrm{CH}, \mathrm{C}-3), 42.1\left(\mathrm{CH}_{2}, \mathrm{C}-5\right)$, $70.1(\mathrm{CH}, \mathrm{C}-4), 71.4(\mathrm{CH}, \mathrm{C}-6), 113.8\left(\mathrm{CH}_{2}, \mathrm{C}-1\right), 125.4(\mathrm{CH}, \mathrm{Ph}), 126.8(\mathrm{CH}, \mathrm{Ph}), 128.2(\mathrm{CH}, \mathrm{Ph})$, $137.2(\mathrm{CH}, \mathrm{C}-2), 145.3$ (quat. C, ipsoPh); $\mathrm{m} / \mathrm{z}$ (TOF ES+) $371.2\left[(\mathrm{M}+\mathrm{Na})^{+}, 100 \%\right] ; \mathrm{HRMS} \mathrm{m} / \mathrm{z}$ (TOF $\mathrm{ES}+) 371.1843\left([\mathrm{M}+\mathrm{Na}]^{+} . \mathrm{C}_{19} \mathrm{H}_{32} \mathrm{NaO}_{2} \mathrm{Si}_{2}\right.$ requires 371.1839).

$\left(3 R^{*}, 4 R^{*}, 6 S^{*}\right)$ oxasilinane $8 a$<smiles>C=C[C@H]1[C@@H](OC)CC(c2ccccc2)O[Si]1(CC)CC</smiles>

$$
\mathrm{C}_{19} \mathrm{H}_{32} \mathrm{O}_{2} \mathrm{Si}_{2}
$$

Exact Mass: 348.19408

Mol. Wt.: 348.62718

C, 65.46; H, 9.25; O, 9.18; Si, $16.11 \%$ 
HPLC: $t_{\mathrm{R}}=70.4 \mathrm{~min} ; v_{\max }(\mathrm{film}) / \mathrm{cm}^{-1} 2956 \mathrm{~s}, 2876 \mathrm{~m}, 1626 \mathrm{w}(\mathrm{C}=\mathrm{C}), 1584 \mathrm{w}, 1564 \mathrm{w}, 1455 \mathrm{w}, 1453 \mathrm{~m}$, $1363 \mathrm{w}, 1252 \mathrm{~m}, 1120 \mathrm{~m}, 1067 \mathrm{~s}, 1050 \mathrm{~m}, 1004 \mathrm{~m}, 963 \mathrm{~m}, 880 \mathrm{~m}, 844 \mathrm{~s}, 802 \mathrm{w}, 787 \mathrm{w}, 727 \mathrm{~m}, 698 \mathrm{~m}, 661 \mathrm{~m}$; $\delta_{\mathrm{H}}(300 \mathrm{MHz}) 0.09\left(9 \mathrm{H}, \mathrm{s}, \mathrm{Si}\left(\mathrm{CH}_{3}\right)_{3}\right), 0.50-0.85\left(4 \mathrm{H}\right.$, stack, OSi $\left.\left(\mathrm{CH}_{2} \mathrm{CH}_{3}\right)_{2}\right), 0.90(3 \mathrm{H}, \mathrm{t}, J 7.5$, $\left.\mathrm{OSi}\left(\mathrm{CH}_{2} \mathrm{CH}_{3}\right)\right), 0.98\left(3 \mathrm{H}, \mathrm{t}, J \mathrm{~J} .9, \mathrm{OSi}\left(\mathrm{CH}_{2} \mathrm{CH}_{3}\right)\right), 1.56\left(1 \mathrm{H}\right.$, app. d, J 13.9, 5- $\left.\mathrm{H}_{\mathrm{a}}\right), 1.80(1 \mathrm{H}$, ddd, J 13.9, 5.0, 2.0, 5- $\left.H_{b}\right), 1.89(1 \mathrm{H}, \mathrm{dd}, J 10.1,2.4,3-H), 4.17-4.26(1 \mathrm{H}, \mathrm{m}, 4-H), 4.78-4.90(2 \mathrm{H} \text {, stack, 1-CH })_{2}$, $5.17(1 \mathrm{H}, \mathrm{dd}, J 11.0,1.6,6-H), 5.84\left(1 \mathrm{H}\right.$, app. dt, J 17.3, 10.1, 2-H), 7.09-7.30 (5H, stack, PhH); $\delta_{\mathrm{C}}(75$ $\mathrm{MHz}) 0.3\left(\mathrm{CH}_{3}, \mathrm{Si}\left(\mathrm{CH}_{3}\right)_{3}\right), 4.8\left(\mathrm{CH}_{2}\right.$, OSi $\left.\left(\mathrm{CH}_{2} \mathrm{CH}_{3}\right)\right), 5.9\left(\mathrm{CH}_{2}, \mathrm{OSi}\left(\mathrm{CH}_{2} \mathrm{CH}_{3}\right)\right), 6.7\left(\mathrm{CH}_{3}, \mathrm{OSi}\left(\mathrm{CH}_{2} \mathrm{CH}_{3}\right)\right)$, $6.8\left(\mathrm{CH}_{3}, \mathrm{OSi}\left(\mathrm{CH}_{2} \mathrm{CH}_{3}\right)\right), 38.3(\mathrm{CH}, \mathrm{C}-3), 45.9\left(\mathrm{CH}_{2}, \mathrm{C}-5\right), 69.5(\mathrm{CH}, \mathrm{C}-4), 71.5(\mathrm{CH}, \mathrm{C}-6), 112.8\left(\mathrm{CH}_{2}\right.$, C-1), $125.3(\mathrm{CH}, \mathrm{Ph}), 126.9(\mathrm{CH}, \mathrm{Ph}), 128.2(\mathrm{CH}, \mathrm{Ph}), 137.9(\mathrm{CH}, \mathrm{C}-2), 145.6$ (quat. C, ipsoPh); $\mathrm{m} / \mathrm{z}$ (TOF ES+) $371.2\left[(\mathrm{M}+\mathrm{Na})^{+}, 100 \%\right]$; HRMS $\mathrm{m} / \mathrm{z}$ (TOF ES+) $371.1837\left([\mathrm{M}+\mathrm{Na}]^{+} . \mathrm{C}_{19} \mathrm{H}_{32} \mathrm{NaO}_{2} \mathrm{Si}_{2}\right.$ requires 371.1839$)$.

$\left(1 S^{*}, 3 R^{*}, 4 S^{*}\right)$ 1-Phenyl-hex-5-ene-1,3,4-triol 9a

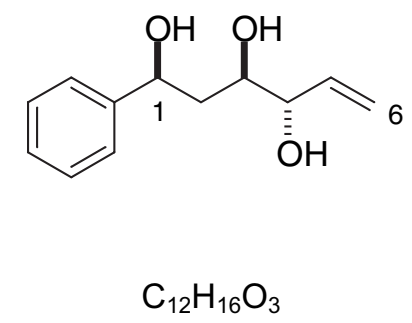

Exact Mass: 208.10995

Mol. Wt.: 208.25364

C, $69.21 ; \mathrm{H}, 7.74 ; \mathrm{O}, 23.05 \%$

$\mathrm{H}_{2} \mathrm{O}_{2}\left(4.63 \mathrm{~g}, 60 \%\right.$ in $\left.\mathrm{H}_{2} \mathrm{O}, 81.8 \mathrm{mmol}\right), \mathrm{KHCO}_{3}(1.23 \mathrm{~g}, 12.3 \mathrm{mmol})$ and $\mathrm{KF}(1.19 \mathrm{~g}, 20.4 \mathrm{mmol})$ were added to a solution of the products from the allylation of aldehyde $6 \mathbf{a}(2.42 \mathrm{~g}, 2.5 \mathrm{mmol}$ of oxasilinane 7a) in MeOH:THF (1:1, $41 \mathrm{~mL})$ and the resulting mixture was stirred for 5 days. Aqueous work-up and purification by flash column chromatography (70 $\rightarrow 80 \%$ EtOAc in hexane) afforded trio/ 9 a as a colorless viscous oil (383 mg, 73\%); $R_{f}=0.25$ (70\% EtOAc in hexane); (Found: C, 69.05; H, 7.91 . $\mathrm{C}_{12} \mathrm{H}_{16} \mathrm{O}_{3}$ requires $\left.\mathrm{C}, 69.21 ; \mathrm{H}, 7.74 \%\right) ; v_{\max }\left(\right.$ film) $/ \mathrm{cm}^{-1} 3380 \mathrm{br}$ vs $(\mathrm{OH}), 3086 \mathrm{~s}, 3030 \mathrm{~s}, 2981 \mathrm{~s}, 2919 \mathrm{~s}$, 1954 w, 1882w, 1723m, 1644m (C=C), 1604w (C=C), 1494s, 1454s, 1434s, 1258s, 1204s, 1063s, 995s, 928s, 853m, 821m, 761s, 701s; $\delta_{\mathrm{H}}(300 \mathrm{MHz}) 1.79\left(1 \mathrm{H}\right.$, app. dt, J 14.7, 3.0, $\left.1 \times 2-\mathrm{CH}_{\mathrm{a}} \mathrm{H}_{\mathrm{b}}\right), 1.80-$ $1.97\left(1 \mathrm{H}, \mathrm{m}, 2-\mathrm{CH}_{\mathrm{a}} \mathrm{H}_{\mathrm{b}}\right), 2.30(1 \mathrm{H}, \mathrm{br} \mathrm{s}, \mathrm{OH}), 3.10(1 \mathrm{H}, \mathrm{br} \mathrm{s}, \mathrm{OH}), 3.50(1 \mathrm{H}, \mathrm{br} \mathrm{s}, \mathrm{OH}), 3.99(1 \mathrm{H}, \mathrm{dt}, J$ 9.9, 3.0, 3-H), 4.12-4.16 (1H, m, 4-H), $4.94\left(1 \mathrm{H}, \mathrm{dd}, J\right.$ 9.9, 3.0, 1-H), $5.24\left(1 \mathrm{H}, \mathrm{d}, J\right.$ 10.7, 6- $\left.H_{c i s}\right), 5.32$ $\left(1 \mathrm{H}, \mathrm{d}, J\right.$ 16.9, 6- $\left.H_{\text {trans }}\right), 5.88(1 \mathrm{H}, \mathrm{ddd}, J 16.9,10.7,6.2,5-H), 7.24-7.41(5 \mathrm{H}$, stack, $\mathrm{PhH}) ; \delta_{\mathrm{C}}(75 \mathrm{MHz})$ $39.5\left(\mathrm{CH}_{2}, \mathrm{C}-2\right), 74.3(\mathrm{CH}, \mathrm{CHOH}), 74.4(\mathrm{CH}, \mathrm{CHOH}), 75.7(\mathrm{CH}, \mathrm{CHOH}), 117.4\left(\mathrm{CH}_{2}, \mathrm{C}-6\right), 125.7(\mathrm{CH}$, $\mathrm{Ph}), 127.6(\mathrm{CH}, \mathrm{Ph}), 128.4(\mathrm{CH}, \mathrm{Ph}), 135.9(\mathrm{CH}, \mathrm{C}-5), 144.1$ (quat. C, ipsoPh); m/z (TOF ES+) 231 $\left[(\mathrm{M}+\mathrm{Na})^{+}, 100 \%\right]$; HRMS $\mathrm{m} / \mathrm{z}(\mathrm{TOF} E S+) 231.0991\left([\mathrm{M}+\mathrm{Na}]^{+} . \mathrm{C}_{12} \mathrm{H}_{16} \mathrm{NaO}_{3}\right.$ requires 231.0997). 


\section{Acetonide protection of triol 9a (and trace 10a)}

$\mathrm{Na}_{2} \mathrm{SO}_{4}(40 \mathrm{mg})$ and $p \mathrm{TsOH} \cdot \mathrm{H}_{2} \mathrm{O}(3 \mathrm{mg}, 16 \mu \mathrm{mol})$ were added to a solution of triol $9 \mathrm{a}$ (30 mg, 0.14 $\mathrm{mmol}$ ) in acetone (non redistilled, $1.4 \mathrm{~mL}$ ) and the resulting mixture was stirred overnight. Aqueous work-up afforded alcohols 11a, 12a and 13a as an inseparable mixture (14:8:1 by ${ }^{1} \mathrm{H}$ NMR) (34 mg, quantitative).

$\left(1 S^{\star}, 3 R^{\star}, 4 S^{\star}\right)$ 2-(2,2-Dimethyl-5-vinyl-[1,3]dioxolan-4-yl)-1-phenyl-ethanol 11a

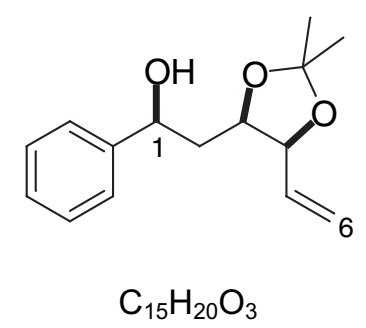

Exact Mass: 248.14125

Mol. Wt.: 248.31750

C, $72.55 ; \mathrm{H}, 8.12 ; \mathrm{O}, 19.33 \%$

$\delta_{\mathrm{H}}\left(\mathrm{C}_{6} \mathrm{D}_{6}, 400 \mathrm{MHz}\right) 1.21\left(3 \mathrm{H}, \mathrm{s},\left(\mathrm{CH}_{3}\right)_{\text {pseudoax. }}\right), 1.43\left(3 \mathrm{H}, \mathrm{s},\left(\mathrm{CH}_{3}\right)_{\text {pseudoeq. }}\right), 1.53(1 \mathrm{H}, \mathrm{ddd}, \mathrm{J}$ 14.0, 4.6, 2.9, $\left.2-\mathrm{CH}_{\mathrm{a}} \mathrm{H}_{\mathrm{b}}\right), 1.93\left(1 \mathrm{H}\right.$, ddd, $\left.J 14.0,10.4,8.7,2-\mathrm{CH}_{\mathrm{a}} \mathrm{H}_{b}\right), 3.15(1 \mathrm{H}, \mathrm{br} \mathrm{s}, 1 \times \mathrm{OH}), 3.97(1 \mathrm{H}, \mathrm{ddd}, J$ 10.4, 6.9, 2.9, 3-H), $4.20(1 \mathrm{H}$, app. t, J 6.9, 4-H), $4.84(1 \mathrm{H}$, dd, J 8.7, 4.6, 1-H), $4.92(1 \mathrm{H}$, ddd, J 10.4, $\left.1.7,1.1,6-H_{\text {cis }}\right), 5.05-5.11\left(1 \mathrm{H}, \mathrm{m}, 6-H_{\text {trans }}\right), 5.52(1 \mathrm{H}, \mathrm{ddd}, J 17.3,10.4,6.9,5-H), 7.07-7.29(3 \mathrm{H}$, stack, $\mathrm{PhH}), 7.44(2 \mathrm{H}, \mathrm{d}, J$ 7.2, oPhH $) ; \delta_{\mathrm{C}}\left(\mathrm{C}_{6} \mathrm{D}_{6}, 100 \mathrm{MHz}\right) 25.6\left(\mathrm{CH}_{3},\left(\mathrm{CH}_{3}\right)_{\text {pseudoax. }}\right), 28.2\left(\mathrm{CH}_{3},\left(\mathrm{CH}_{3}\right)_{\text {pseudoeq. }}\right)$, $40.8\left(\mathrm{CH}_{2}, \mathrm{C}-2\right), 73.6(\mathrm{CH}, \mathrm{C}-1), 77.8(\mathrm{CH}, \mathrm{C}-3), 79.7(\mathrm{CH}, \mathrm{C}-4), 108.8$ (quat. $\left.\mathrm{C}, \mathrm{C}\left(\mathrm{CH}_{3}\right)_{2}\right), 117.6\left(\mathrm{CH}_{2}\right.$, C-6), $126.25(\mathrm{CH}, o \mathrm{Ph}), 126.7-131.1\left(\mathrm{CH}\right.$ and $\mathrm{CD}$, stack, $\left.\mathrm{Ph}, \mathrm{C}_{6} \mathrm{D}_{6}\right), 134.6(\mathrm{CH}, \mathrm{C}-5), 145.2$ (quat. $\mathrm{C}$, ipsoPh).

$\left(1 S^{*}, 3 R^{*}, 4 S^{*}\right)$ 1-(2,2-Dimethyl-6-phenyl-[1,3]dioxan-4-yl)-prop-2-en-1-ol 12a

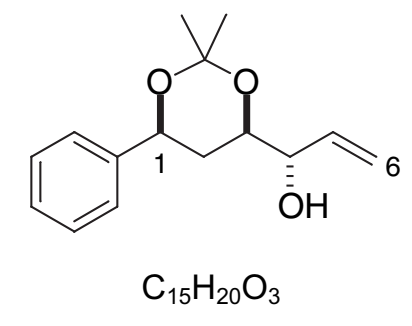

Exact Mass: 248.14125

Mol. Wt.: 248.31750

C, $72.55 ; \mathrm{H}, 8.12 ; \mathrm{O}, 19.33 \%$ 
$\delta_{\mathrm{H}}\left(\mathrm{C}_{6} \mathrm{D}_{6}, 400 \mathrm{MHz}\right) 1.29\left(3 \mathrm{H}, \mathrm{s},\left(\mathrm{CH}_{3}\right)_{\text {pseudoax }}\right), 1.46-1.50\left(1 \mathrm{H}, \mathrm{m}, 2-\mathrm{CH}_{\mathrm{a}} \mathrm{H}_{\mathrm{b}}\right), 1.51\left(3 \mathrm{H}, \mathrm{s},\left(\mathrm{CH}_{3}\right)_{\text {pseudoeq. }}\right)$, $1.70\left(1 \mathrm{H}\right.$, app. q, J 12.0, 2- $\left.\mathrm{CH}_{\mathrm{a}} H_{b}\right), 2.01(1 \mathrm{H}, \mathrm{br} \mathrm{s}, \mathrm{OH}), 3.73(1 \mathrm{H}, \mathrm{ddd}, J 12.0,4.1,2.5,3-H), 4.07-4.12$ $(1 \mathrm{H}, \mathrm{m}, 4-H), 4.65(1 \mathrm{H}, \mathrm{dd}, J 12.0,2.7,1-H), 5.05-5.11\left(1 \mathrm{H}, \mathrm{m}, 6-H_{\text {cis }}\right), 5.35(1 \mathrm{H}$, app. dt, J 17.3, 1.9, 6$\left.H_{\text {trans }}\right), 5.79(1 \mathrm{H}$, ddd, J 17.3, 10.7, 5.2, 5-H), 7.07-7.29 (3H, stack, $\mathrm{PhH}), 7.34(2 \mathrm{H}, \mathrm{d}, J$ 7.2, oPhH); $\delta_{\mathrm{C}}\left(\mathrm{C}_{6} \mathrm{D}_{6}, 100 \mathrm{MHz}\right) 19.8\left(\mathrm{CH}_{3},\left(\mathrm{CH}_{3}\right)_{\text {pseudoax. }}\right), 30.3\left(\mathrm{CH}_{3},\left(\mathrm{CH}_{3}\right)_{\text {pseudoeq. }}\right), 33.3\left(\mathrm{CH}_{2}, \mathrm{C}-2\right), 71.2(\mathrm{CH}, \mathrm{C}-$ 1), $72.5(\mathrm{CH}, \mathrm{C}-3), 74.3(\mathrm{CH}, \mathrm{C}-4), 99.1$ (quat. $\left.\mathrm{C}, \mathrm{C}\left(\mathrm{CH}_{3}\right)_{2}\right), 116.0\left(\mathrm{CH}_{2}, \mathrm{C}-6\right), 126.17(\mathrm{CH}, \mathrm{oPh})$, 126.7-131.1 ( $\mathrm{CH}$ and $\mathrm{CD}$, stack, $\left.\mathrm{Ph}, \mathrm{C}_{6} \mathrm{D}_{6}\right), 136.7(\mathrm{CH}, \mathrm{C}-5), 143.1$ (quat. $\mathrm{C}$, ipsoPh).

$\left(1 S^{*}, 3 R^{*}, 4 R^{*}\right)$ 2-(2,2-Dimethyl-5-vinyl-[1,3]dioxolan-4-yl)-1-phenyl-ethanol 13a<smiles>CC1(C)OC(C=O)C(CC(O)(I)c2ccccc2)O1</smiles>

$$
\mathrm{C}_{15} \mathrm{H}_{20} \mathrm{O}_{3}
$$

Exact Mass: 248.14125

Mol. Wt.: 248.31750

C, $72.55 ; \mathrm{H}, 8.12 ; \mathrm{O}, 19.33 \%$

$\delta_{\mathrm{H}}\left(\mathrm{C}_{6} \mathrm{D}_{6}, 400 \mathrm{MHz}\right)$-identifiable resonances- $1.37\left(3 \mathrm{H}, \mathrm{s}, \mathrm{CH}_{3}\right), 1.38\left(3 \mathrm{H}, \mathrm{s}, \mathrm{CH}_{3}\right), 1.82-1.88(1 \mathrm{H}, \mathrm{m}, 2-$ $\left.\mathrm{CH}_{a} \mathrm{H}_{\mathrm{b}}\right)$, 3.93-3.98 $\left(1 \mathrm{H}\right.$, stack, 4-H), $4.96\left(1 \mathrm{H}\right.$, br d (+ unresolved fine coupling), J 10.5, 6- $\left.H_{c i s}\right), 5.16$ $\left(1 \mathrm{H}, \mathrm{dm}, J 16.9,6-H_{\text {trans }}\right), 5.66\left(1 \mathrm{H}\right.$, ddd, J 16.9, 10.5, 6.7, 5-H); $\delta_{\mathrm{C}}\left(\mathrm{C}_{6} \mathrm{D}_{6}, 100 \mathrm{MHz}\right) 27.1\left(\mathrm{CH}_{3}, \mathrm{CH}_{3}\right)$, $27.5\left(\mathrm{CH}_{3}, \mathrm{CH}_{3}\right), 41.3\left(\mathrm{CH}_{2}, \mathrm{C}-2\right), 77.9(\mathrm{CH}, \mathrm{C}-3), 82.7(\mathrm{CH}, \mathrm{C}-4), 109.0$ (quat. C, $\left.\mathrm{C}\left(\mathrm{CH}_{3}\right)_{2}\right), 117.9$ $\left(\mathrm{CH}_{2}, \mathrm{C}-6\right), 125.9(\mathrm{CH}, \mathrm{oPh}), 135.7(\mathrm{CH}, \mathrm{C}-5), 145.6$ (quat. C, ipsoPh).

\section{Allylation reaction of Aldehyde 6b:}

TMSOTf ( $324 \mu \mathrm{L}, 1.8 \mathrm{mmol})$ was added to a solution of aldehyde $6 \mathbf{b}(607 \mathrm{mg}, 1.8 \mathrm{mmol})$ and 2,6DTBMP (443 mg, $2.2 \mathrm{mmol})$ in $\mathrm{CH}_{2} \mathrm{Cl}_{2}(18 \mathrm{~mL})$ at $-78{ }^{\circ} \mathrm{C}$ and the reaction mixture was stirred for $8 \mathrm{~h}$. Aqueous work-up and removal of the solvent afforded compounds $\mathbf{7 b}$ and $\mathbf{8} \mathbf{b}$ (69:17; inseparable mixture) as a yellow liquid ( $1.003 \mathrm{~g}, 86 \%$, resulting mass being diene products). This was used in the following step without any further purification. Analytically pure samples of compounds $\mathbf{7 b}$ and $\mathbf{8 b}$ were obtained as colorless oils by preparative $\operatorname{HPLC}\left(5 \% \mathrm{H}_{2} \mathrm{O}\right.$ in $\left.\mathrm{MeCN}\right) ; \mathbf{7 b} t_{\mathrm{R}}=35.6 \mathrm{~min}$ and $\mathbf{8 b} t_{\mathrm{R}}=43.0$ $\min$. 
<smiles>C=CC1[C@H](OC)C[C@@H](c2ccco2)O[Si]1(CC)CC</smiles>

$$
\mathrm{C}_{17} \mathrm{H}_{30} \mathrm{O}_{3} \mathrm{Si}_{2}
$$

Exact Mass: 338.17335

Mol. Wt.: 338.58930

C, $60.30 ; \mathrm{H}, 8.93 ; \mathrm{O}, 14.18 ; \mathrm{Si}, 16.59 \%$

HPLC: $t_{\mathrm{R}}=35.6 \mathrm{~min} ; v_{\max }(\mathrm{film}) / \mathrm{cm}^{-1} 3077 \mathrm{w}, 2956 \mathrm{~s}, 2878 \mathrm{~m}, 1628 \mathrm{w}(\mathrm{C}=\mathrm{C}), 1505 \mathrm{w}, 1460 \mathrm{w}, 1414 \mathrm{w}$, $1351 \mathrm{w}, 1252 \mathrm{~s}, 1156 \mathrm{~m}, 1070 \mathrm{~s}, 1005 \mathrm{~m}, 956 \mathrm{w}, 876 \mathrm{~m}, 840 \mathrm{~s}, 727 \mathrm{~m}, 628 \mathrm{~m} ; \delta_{\mathrm{H}}(400 \mathrm{MHz}) 0.10(9 \mathrm{H}, \mathrm{s}$, $\left.\mathrm{Si}\left(\mathrm{CH}_{3}\right)_{3}\right), 0.55\left(2 \mathrm{H}, \mathrm{q}, J 7.8, \mathrm{OSi}\left(\mathrm{CH}_{2} \mathrm{CH}_{3}\right)\right), 0.76\left(2 \mathrm{H}, \mathrm{q}, J 7.8, \mathrm{OSi}\left(\mathrm{CH}_{2} \mathrm{CH}_{3}\right)\right), 0.86(3 \mathrm{H}, \mathrm{t}, J 7.8$, $\left.\mathrm{OSi}\left(\mathrm{CH}_{2} \mathrm{CH}_{3}\right)\right), 1.01\left(3 \mathrm{H}, \mathrm{t}, J 7.8\right.$, OSi $\left.\left(\mathrm{CH}_{2} \mathrm{CH}_{3}\right)\right), 1.90\left(1 \mathrm{H}\right.$, ddd, J 14.1, 8.1, 4.0, 5- $\left.\mathrm{CH}_{\mathrm{a}} \mathrm{H}_{\mathrm{b}}\right), 1.98(1 \mathrm{H}$, dd, J 10.3, 7.3, 3-H), 2.20 (1H, ddd, J 14.1, 6.6, 2.4, 5- $\mathrm{CH}_{\mathrm{a}} H_{b}$ ), 4.18 (1H, app. td, J 7.7, 2.2, 4-H), 4.90$4.98\left(2 \mathrm{H}\right.$, stack, 1- $\left.\left.\mathrm{CH}_{2}\right), 5.23(1 \mathrm{H}, \mathrm{dd}, J 6.5,4.0,6-H)\right), 5.74(1 \mathrm{H}, \mathrm{dt}, J 16.9,10.4,2-H), 6.22(1 \mathrm{H}, \mathrm{d}, J$ 3.2, 8-H), $6.32\left(1 \mathrm{H}\right.$, dd, J 3.2, 2.0, 9-H), $7.36(1 \mathrm{H}, \mathrm{dd}, J 2.0,0.7,10-H) ; \delta_{\mathrm{C}}(100 \mathrm{MHz}) 0.1\left(\mathrm{CH}_{3}\right.$, $\left.\mathrm{Si}\left(\mathrm{CH}_{3}\right)_{3}\right), 5.1\left(\mathrm{CH}_{2}, \mathrm{OSi}\left(\mathrm{CH}_{2} \mathrm{CH}_{3}\right)\right), 5.5\left(\mathrm{CH}_{2}, \mathrm{OSi}\left(\mathrm{CH}_{2} \mathrm{CH}_{3}\right)\right), 6.1\left(\mathrm{CH}_{3}, \mathrm{OSi}\left(\mathrm{CH}_{2} \mathrm{CH}_{3}\right)\right), 6.5\left(\mathrm{CH}_{3}\right.$, OSi $\left.\left(\mathrm{CH}_{2} \mathrm{CH}_{3}\right)\right), 39.1\left(\mathrm{CH}_{2}, \mathrm{C}-5\right), 40.1(\mathrm{CH}, \mathrm{C}-3), 66.0(\mathrm{CH}, \mathrm{C}-6), 70.1(\mathrm{CH}, \mathrm{C}-4), 105.6(\mathrm{CH}, \mathrm{C}-8)$, $110.1(\mathrm{CH}, \mathrm{C}-9), 114.0\left(\mathrm{CH}_{2}, \mathrm{C}-1\right), 137.1(\mathrm{CH}, \mathrm{C}-2), 141.5(\mathrm{CH}, \mathrm{C}-10), 156.9$ (quat. C, C-7); $\mathrm{m} / \mathrm{z}$ (TOF ES+) $361.2\left[(\mathrm{M}+\mathrm{Na})^{+}, 100 \%\right]$; HRMS m/z (TOF ES+) $361.1627\left([\mathrm{M}+\mathrm{Na}]^{+} . \mathrm{C}_{17} \mathrm{H}_{30} \mathrm{NaO}_{3} \mathrm{Si}_{2}\right.$ requires 361.1631).

\section{$\left(3 R^{\star}, 4 R^{\star}, 6 S^{*}\right)$ oxasilinane $8 \mathrm{~b}$}<smiles>C=C[C@H]1[C@@H](OC)C[C@@H](c2ccco2)O[Si]1(CC)CC</smiles>

$$
\mathrm{C}_{17} \mathrm{H}_{30} \mathrm{O}_{3} \mathrm{Si}_{2}
$$

Exact Mass: 338.17335

Mol. Wt.: 338.58930

C, $60.30 ; \mathrm{H}, 8.93 ; \mathrm{O}, 14.18 ; \mathrm{Si}, 16.59 \%$

HPLC: $t_{\mathrm{R}}=43.0 \mathrm{~min} ; v_{\max }(\mathrm{film}) / \mathrm{cm}^{-1} 2956 \mathrm{~s}, 2877 \mathrm{~m}, 1626 \mathrm{w}(\mathrm{C}=\mathrm{C}), 1460 \mathrm{w}, 1350 \mathrm{w}, 1322 \mathrm{w}, 1252 \mathrm{~m}$, $1150 \mathrm{w}, 1117 \mathrm{~m}, 1073 \mathrm{~m}, 1049 \mathrm{~m}, 1004 \mathrm{w}, 963 \mathrm{w}, 880 \mathrm{w}, 840 \mathrm{~m}, 806 \mathrm{w}, 784 \mathrm{w}, 727 \mathrm{~m} ; \delta_{\mathrm{H}}(400 \mathrm{MHz}) 0.13$ 
$\left(9 \mathrm{H}, \mathrm{s}, \mathrm{Si}\left(\mathrm{CH}_{3}\right)_{3}\right), 0.53-0.92\left(4 \mathrm{H}\right.$, stack, OSi $\left.\left(\mathrm{CH}_{2} \mathrm{CH}_{3}\right)_{2}\right), 0.98\left(3 \mathrm{H}, \mathrm{t}, J 7.8, \mathrm{OSi}\left(\mathrm{CH}_{2} \mathrm{CH}_{3}\right)\right), 0.99(3 \mathrm{H}, \mathrm{t}, J$ 7.8, OSi $\left.\left(\mathrm{CH}_{2} \mathrm{CH}_{3}\right)\right)$, 1.83-1.99 (3H, stack, 3- $\left.\mathrm{H}, 5-\mathrm{CH}_{2}\right), 4.28-4.34(1 \mathrm{H}, \mathrm{m}, 4-\mathrm{H}), 4.89(1 \mathrm{H}, \mathrm{dd}, J 10.2$, 2.2, $\left.1-H_{\text {cis }}\right), 4.92\left(1 \mathrm{H}, \mathrm{dd}, J 17.2,1.5,1-H_{\text {trans }}\right), 5.24(1 \mathrm{H}, \mathrm{dd}, J 10.6,2.8,6-H), 5.89(1 \mathrm{H}, \mathrm{dt}, J 17.2$, $10.2,2-H), 6.20(1 \mathrm{H}, \mathrm{d}, J 3.2,8-H), 6.30(1 \mathrm{H}, \mathrm{dd}, J 3.2,2.0,9-H), 7.33-7.37(1 \mathrm{H}, \mathrm{m}, 10-H) ; \delta_{\mathrm{C}}(100$ $\mathrm{MHz}) 0.2\left(\mathrm{CH}_{3}, \mathrm{Si}\left(\mathrm{CH}_{3}\right)_{3}\right), 4.8\left(\mathrm{CH}_{2}\right.$, OSi $\left.\left(\mathrm{CH}_{2} \mathrm{CH}_{3}\right)\right), 5.7\left(\mathrm{CH}_{2}, \mathrm{OSi}\left(\mathrm{CH}_{2} \mathrm{CH}_{3}\right)\right), 6.5\left(\mathrm{CH}_{3}, \mathrm{OSi}\left(\mathrm{CH}_{2} \mathrm{CH}_{3}\right)\right)$, $6.7\left(\mathrm{CH}_{3}, \mathrm{OSi}\left(\mathrm{CH}_{2} \mathrm{CH}_{3}\right)\right), 38.4(\mathrm{CH}, \mathrm{C}-3), 41.2\left(\mathrm{CH}_{2}, \mathrm{C}-5\right), 63.6(\mathrm{CH}, \mathrm{C}-6), 71.1(\mathrm{CH}, \mathrm{C}-4), 105.0(\mathrm{CH}$, C-8), $110.0(\mathrm{CH}, \mathrm{C}-9), 113.0\left(\mathrm{CH}_{2}, \mathrm{C}-1\right), 137.8(\mathrm{CH}, \mathrm{C}-2), 141.5(\mathrm{CH}, \mathrm{C}-10), 157.6$ (quat. C, C-7); m/z (TOF ES + ) $361.2\left[(\mathrm{M}+\mathrm{Na})^{+}, 100 \%\right]$; HRMS $\mathrm{m} / \mathrm{z}(\mathrm{TOF} \mathrm{ES}+) 361.1636\left([\mathrm{M}+\mathrm{Na}]^{+} . \mathrm{C}_{17} \mathrm{H}_{30} \mathrm{NaO}_{3} \mathrm{Si}_{2}\right.$ requires 361.1631$)$.

\title{
$\left(1 S^{*}, 3 R^{*}, 4 S^{*}\right)$ 1-Furan-2-yl-hex-5-ene-1,3,4-triol 9b
}

\author{
$\underbrace{}_{4^{\prime}}$ \\ $\mathrm{C}_{10} \mathrm{H}_{14} \mathrm{O}_{4}$ \\ Exact Mass: 198.08921 \\ Mol. Wt.: 198.21576 \\ C, $60.59 ; \mathrm{H}, 7.12 ; \mathrm{O}, 32.29 \%$
}

$\mathrm{H}_{2} \mathrm{O}_{2}\left(3.06 \mathrm{~g}, 60 \%\right.$ in $\left.\mathrm{H}_{2} \mathrm{O}, 54.0 \mathrm{mmol}\right), \mathrm{KHCO}_{3}(811 \mathrm{mg}, 8.1 \mathrm{mmol})$ and $\mathrm{KF}(784 \mathrm{mg}, 15.5 \mathrm{mmol})$ were added to a solution of the products from the allylation of aldehyde $6 \mathbf{b}(1.578 \mathrm{~g}, 1.61 \mathrm{mmol}$ of oxasilinane $7 \mathbf{b})$ in MeOH:THF $(1: 1,27 \mathrm{~mL})$ and the resulting mixture was stirred for 5 days. Aqueous work-up and purification by flash column chromatography (70 $\rightarrow 90 \%$ EtOAc in hexane) afforded triol 9b as a yellow viscous oil (201 mg, 61\%); $R_{f}=0.27$ (70\% EtOAc in hexane); (Found: C, 60.81; $\mathrm{H}$, 7.16. $\mathrm{C}_{10} \mathrm{H}_{14} \mathrm{O}_{4}$ requires $\left.\mathrm{C}, 60.59 ; \mathrm{H}, 7.12 \%\right) ; v_{\max }($ film $) / \mathrm{cm}^{-1} 3346 \mathrm{br} \mathrm{s}(\mathrm{OH}), 2918 \mathrm{~m}, 1706 \mathrm{w}, 1640 \mathrm{w}$, $1504 \mathrm{~m}, 1425 \mathrm{~m}, 1314 \mathrm{~m}, 1229 \mathrm{~m}, 1173 \mathrm{w}, 1146 \mathrm{~m}, 1068 \mathrm{~s}, 1009 \mathrm{~s}, 929 \mathrm{~m}, 884 \mathrm{~m}, 854 \mathrm{w}, 816 \mathrm{w}$ and $742 \mathrm{~s}$; $\delta_{\mathrm{H}}(300 \mathrm{MHz})$ 1.88-2.08 $\left(2 \mathrm{H}\right.$, stack, $\left.\mathrm{CH}_{2}\right), 3.10(1 \mathrm{H}, \mathrm{br} \mathrm{s}, \mathrm{OH}), 3.82(1 \mathrm{H}, \mathrm{br} \mathrm{s}, \mathrm{OH}), 3.87-3.98(2 \mathrm{H}$, stack, $\mathrm{CHOH}, \mathrm{OH}), 4.11-4.19(1 \mathrm{H}, \mathrm{m}, \mathrm{CHOH}), 4.90(1 \mathrm{H}, \mathrm{dd}, J$ 9.2, 4.1, $\mathrm{CHOH}), 5.23(1 \mathrm{H}, \mathrm{d}, J 10.3$, $\left.=\mathrm{CH}_{\text {cis }}\right), 5.32\left(1 \mathrm{H}, \mathrm{d}, \mathrm{J} 17.3,=\mathrm{CH} H_{\text {trans }}\right), 5.87\left(1 \mathrm{H}, \mathrm{ddd}, \mathrm{J} 17.3,10.3,6.2, \mathrm{CH}=\mathrm{CH}_{2}\right), 6.22(1 \mathrm{H}, \mathrm{d}, J 2.9$, 2'-H), 6.29-6.32 (1H, m, 3'-H), $7.34\left(1 \mathrm{H}\right.$, app. s, 4'-H); $\delta_{\mathrm{C}}(75 \mathrm{MHz}) 35.9\left(\mathrm{CH}_{2}\right), 67.5(\mathrm{CH}, \mathrm{CHOH}), 73.9$ $(\mathrm{CH}, \mathrm{CHOH}), 75.6(\mathrm{CH}, \mathrm{CHOH}), 105.9\left(\mathrm{CH}, \mathrm{C}-2^{\prime}\right.$ or $\left.\mathrm{C}-3^{\prime}\right), 110.2\left(\mathrm{CH}, \mathrm{C}-3^{\prime}\right.$ or $\left.\mathrm{C}-2^{\prime}\right), 117.6\left(\mathrm{CH}_{2},=\mathrm{CH}_{2}\right)$, $135.9(\mathrm{CH},=\mathrm{CH}), 142.0\left(\mathrm{CH}, \mathrm{C}-4^{\prime}\right)$ and 155.9 (quat. $\left.\mathrm{C}, \mathrm{C}-1^{\prime}\right) ; \mathrm{m} / \mathrm{z}$ (TOF ES+) $221.1\left([\mathrm{M}+\mathrm{Na}]^{+}, 100 \%\right)$; HRMS $\mathrm{m} / \mathrm{z}$ (TOF ES+) $221.0786\left([\mathrm{M}+\mathrm{Na}]^{+} . \mathrm{C}_{10} \mathrm{H}_{14} \mathrm{NaO}_{4}\right.$ requires 221.0790).

\section{Allylation reaction of Aldehyde 6c:}

TMSOTf (145 $\mu \mathrm{L}, 0.80 \mathrm{mmol})$ was added to a solution of aldehyde $6 \mathrm{c}(297 \mathrm{mg}, 0.80 \mathrm{mmol})$ and 2,6DTBMP $(197 \mathrm{mg}, 0.96 \mathrm{mmol})$ in $\mathrm{CH}_{2} \mathrm{Cl}_{2}(8 \mathrm{~mL})$ at $-78{ }^{\circ} \mathrm{C}$ and the reaction mixture was stirred for $8 \mathrm{~h}$. 
Aqueous work-up and removal of the solvent afforded compounds $7 \mathrm{c}$ and $\mathbf{8 c}$ (64:18; inseparable mixture) as a yellow liquid (524 $\mathrm{mg}, 82 \%$, remaining mass being diene products). This was used in the following step without any further purification. Analytically pure samples of compounds $7 \mathrm{c}$ and $\mathbf{8 c}$ were obtained as colorless oils by preparative HPLC $\left(3 \% \mathrm{H}_{2} \mathrm{O}\right.$ in MeCN); $7 \mathbf{c} t_{\mathrm{R}}=48.3 \mathrm{~min}$ and $8 \mathrm{c} t_{\mathrm{R}}=83.9$ $\min$.

$\left(3 S^{\star}, 4 R^{\star}, 6 S^{\star}, 7 E\right)$ oxasilinane $7 \mathrm{c}$

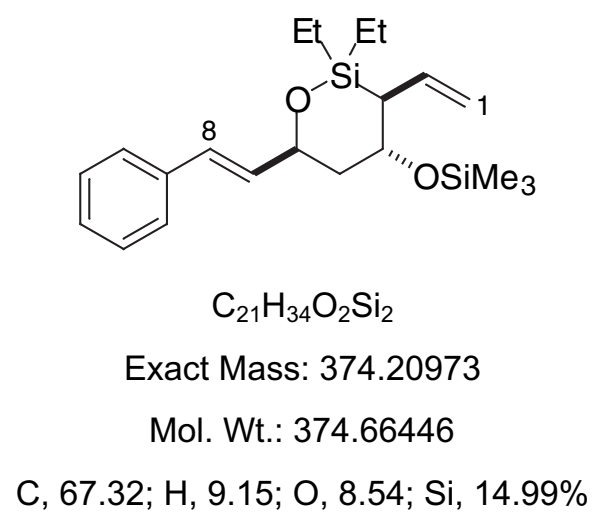

HPLC: $t_{\mathrm{R}}=48.3 \mathrm{~min} ; v_{\max }(\mathrm{film}) / \mathrm{cm}^{-1} 3079 \mathrm{w}, 3040 \mathrm{w}, 3027 \mathrm{w}, 2956 \mathrm{~s}, 2915 \mathrm{~m}, 2876 \mathrm{~s}, 1942 \mathrm{w}, 1872 \mathrm{w}$, 1798w, 1739, 1627m (C=C), 1600w, 1578w, 1496m, 1459m, 1448m, 1414m, 1364m, 1340m, 1328m, $1303 \mathrm{w}, 1251 \mathrm{~s}, 1201 \mathrm{w}, 1159 \mathrm{~m}, 1120 \mathrm{~m}, 1071 \mathrm{~s}, 998 \mathrm{~s}, 967 \mathrm{~m}, 942 \mathrm{~m}, 885 \mathrm{~m}, 869 \mathrm{~m}, 842 \mathrm{~s}, 780 \mathrm{~m}, 745 \mathrm{~s}$, $725 \mathrm{~m}, 692 \mathrm{~m}, 658 \mathrm{w}, 626 \mathrm{~m} ; \delta_{\mathrm{H}}(500 \mathrm{MHz}) 0.12\left(9 \mathrm{H}, \mathrm{s}, \mathrm{OSi}\left(\mathrm{CH}_{3}\right)_{3}\right), 0.61-0.71\left(2 \mathrm{H}, \mathrm{m}, \mathrm{OSi}\left(\mathrm{CH}_{2} \mathrm{CH}_{3}\right)\right)$, 0.76-0.82 (2H, m, OSi $\left.\left(\mathrm{CH}_{2} \mathrm{CH}_{3}\right)\right), 0.99\left(3 \mathrm{H}, \mathrm{t}, J\right.$ 8.0, OSi $\left.\left(\mathrm{CH}_{2} \mathrm{CH}_{3}\right)\right), 1.03\left(3 \mathrm{H}, \mathrm{t}, J 7.5, \mathrm{OSi}\left(\mathrm{CH}_{2} \mathrm{CH}_{3}\right)\right)$, $1.81\left(1 \mathrm{H}\right.$, ddd, J 14.0, 7.3, 3.7, 5- $\left.\mathrm{CH}_{\mathrm{a}} \mathrm{H}_{\mathrm{b}}\right), 1.91\left(1 \mathrm{H}, \mathrm{ddd}, J 14.0,7.3,2.1,5-\mathrm{CH}_{\mathrm{a}} \mathrm{H}_{\mathrm{b}}\right), 1.97(1 \mathrm{H}, \mathrm{dd}, J$ 10.3, 6.8, 3-H), $4.13\left(1 \mathrm{H}\right.$, app. t, J 6.9, 4-H), 4.83-4.88 (1H, m, 6-H), $4.93\left(1 \mathrm{H}, \mathrm{d}, J 10.3,1-H_{\text {cis }}\right), 4.95$ $\left(1 \mathrm{H}, \mathrm{d}, J 17.5,1-H_{\text {trans }}\right), 5.75(1 \mathrm{H}, \mathrm{dt}, J 17.5,10.3,2-H), 6.26(1 \mathrm{H}, \mathrm{dd}, J 15.9,5.4,7-H), 6.60(1 \mathrm{H}, \mathrm{d}, J$ 15.9, 8-H), $7.22(1 \mathrm{H}, \mathrm{t}, J 7.5, p \mathrm{PhH}), 7.31(2 \mathrm{H}, \mathrm{t}, J 7.5,2 \times m \mathrm{PhH}), 7.37(2 \mathrm{H}, \mathrm{d}, J 7.5,2 \times o \mathrm{PhH})$; $\delta_{\mathrm{C}}(125 \mathrm{MHz}) 0.2\left(\mathrm{CH}_{3}, \mathrm{Si}\left(\mathrm{CH}_{3}\right)_{3}\right), 5.4\left(\mathrm{CH}_{2}, \mathrm{OSi}\left(\mathrm{CH}_{2} \mathrm{CH}_{3}\right)\right), 5.7\left(\mathrm{CH}_{2}, \mathrm{OSi}\left(\mathrm{CH}_{2} \mathrm{CH}_{3}\right)\right), 6.0\left(\mathrm{CH}_{3}\right.$, OSi $\left.\left(\mathrm{CH}_{2} \mathrm{CH}_{3}\right)\right)$, $6.6\left(\mathrm{CH}_{3}, \mathrm{OSi}\left(\mathrm{CH}_{2} \mathrm{CH}_{3}\right)\right), 39.8(\mathrm{CH}, \mathrm{C}-3), 40.8\left(\mathrm{CH}_{2}, \mathrm{C}-5\right), 70.1(\mathrm{CH}, \mathrm{C}-6), 70.3(\mathrm{CH}, \mathrm{C}-$ 4), $113.9\left(\mathrm{CH}_{2}, \mathrm{C}-1\right), 126.4(\mathrm{CH}, o \mathrm{Ph}), 127.3(\mathrm{CH}, p \mathrm{Ph}), 128.5(\mathrm{CH}, m \mathrm{Ph}), 129.1(\mathrm{CH}, \mathrm{C}-8), 132.5$ (CH, C-7), 137.1 (quat. C, ipsoPh), 137.2 (CH, C-2); m/z (TOF ES+) $397.0\left([\mathrm{M}+\mathrm{Na}]^{+}, 100 \%\right.$ ); HRMS $\mathrm{m} / z$ (TOF ES+) $397.2005\left([\mathrm{M}+\mathrm{Na}]^{+} . \mathrm{C}_{21} \mathrm{H}_{34} \mathrm{NaO}_{2} \mathrm{Si}_{2}\right.$ requires 397.1995). 


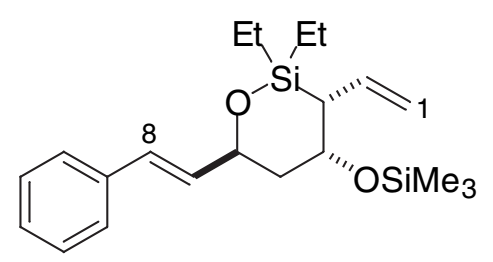

$$
\mathrm{C}_{21} \mathrm{H}_{34} \mathrm{O}_{2} \mathrm{Si}_{2}
$$

Exact Mass: 374.20973

Mol. Wt.: 374.66446

C, $67.32 ; \mathrm{H}, 9.15 ; \mathrm{O}, 8.54 ; \mathrm{Si}, 14.99 \%$

HPLC: $t_{\mathrm{R}}=83.9 \mathrm{~min} ; v_{\max }(\mathrm{film}) / \mathrm{cm}^{-1} 3077 \mathrm{w}, 3027 \mathrm{w}, 2956 \mathrm{~s}, 2913 \mathrm{~s}, 2876 \mathrm{~s}, 1626 \mathrm{w}(\mathrm{C}=\mathrm{C}), 1601 \mathrm{w}$, 1495w, 1449w, 1416w, 1362w, 1341w, 1321w, 1301w, 1252s, 1120w, 1170w, 1140m, 1088s, 1057s, $1003 \mathrm{~m}, 962 \mathrm{~s}, 899 \mathrm{~m}, 868 \mathrm{~m}, 841 \mathrm{~s}, 809 \mathrm{w}, 777 \mathrm{w}, 743 \mathrm{~m}, 722 \mathrm{~m}, 692 \mathrm{~m}, 666 \mathrm{~m} ; \delta_{\mathrm{H}}(400 \mathrm{MHz}) 0.14(9 \mathrm{H}, \mathrm{s}$, OSi $\left.\left(\mathrm{CH}_{3}\right)_{3}\right), 0.54-0.91\left(4 \mathrm{H}\right.$, stack, OSi $\left.\left(\mathrm{CH}_{2} \mathrm{CH}_{3}\right)_{2}\right), 0.99\left(3 \mathrm{H}, \mathrm{t}, J 7.8, \mathrm{OSi}\left(\mathrm{CH}_{2} \mathrm{CH}_{3}\right)\right), 1.02(3 \mathrm{H}, \mathrm{t}, J 8.0$, OSi $\left.\left(\mathrm{CH}_{2} \mathrm{CH}_{3}\right)\right), 1.55\left(1 \mathrm{H}, \mathrm{dd}, J 13.9,11.7,5-H_{\text {pseudoax. }}\right), 1.80\left(1 \mathrm{H}, \mathrm{ddd}, J 13.9,5.1,2.2,5-H_{\text {pseudoeq. }}\right)$, $1.90(1 \mathrm{H}, \mathrm{dd}, J$ 10.0, 2.7, 3-H), 4.25-4.31 (1H, m, 4-H), 4.81-4.87 (1H, m, 6-H), $4.87(1 \mathrm{H}, \mathrm{d}, J$ 10.3, 1$\left.H_{\text {cis }}\right), 4.91\left(1 \mathrm{H}, \mathrm{d}, J\right.$ 16.5, 1- $\left.H_{\text {trans }}\right), 5.90(1 \mathrm{H}, \mathrm{dt}, J$ 16.5, 10.3, 2-H), $6.22(1 \mathrm{H}, \mathrm{dd}, J 15.7,5.7,7-H), 5.59$ $(1 \mathrm{H}, \mathrm{d}, J$ 15.7, 8-H), $7.19(1 \mathrm{H}, \mathrm{t}, J$ 7.3, pPhH), $7.27(2 \mathrm{H}, \mathrm{t}, J$ 7.3, $2 \times \mathrm{mPhH}), 7.37(2 \mathrm{H}, \mathrm{d}, J 7.3,2 \times$ $o \mathrm{PhH}) ; \delta_{\mathrm{C}}(100 \mathrm{MHz}) 0.2\left(\mathrm{CH}_{3}, \mathrm{Si}\left(\mathrm{CH}_{3}\right)_{3}\right), 4.9\left(\mathrm{CH}_{2}, \mathrm{OSi}\left(\mathrm{CH}_{2} \mathrm{CH}_{3}\right)\right), 5.7\left(\mathrm{CH}_{2}, \mathrm{OSi}\left(\mathrm{CH}_{2} \mathrm{CH}_{3}\right)\right), 6.6\left(\mathrm{CH}_{3}\right.$, OSi $\left.\left(\mathrm{CH}_{2} \mathrm{CH}_{3}\right)\right), 6.7\left(\mathrm{CH}_{3}\right.$, OSi $\left.\left(\mathrm{CH}_{2} \mathrm{CH}_{3}\right)\right), 38.5(\mathrm{CH}, \mathrm{C}-3), 43.1\left(\mathrm{CH}_{2}, \mathrm{C}-5\right), 68.0(\mathrm{CH}, \mathrm{C}-6), 71.3(\mathrm{CH}, \mathrm{C}-$ 4), $112.8\left(\mathrm{CH}_{2}, \mathrm{C}-1\right), 126.4(\mathrm{CH}, \mathrm{oPh}), 127.2(\mathrm{CH}, p \mathrm{Ph}), 128.4(\mathrm{CH}, m \mathrm{Ph}), 128.5(\mathrm{CH}, \mathrm{C}-8), 133.1$ ( $\mathrm{CH}, \mathrm{C}-7), 137.2$ (quat. C, ipsoPh), $137.9(\mathrm{CH}, \mathrm{C}-2) ; \mathrm{m} / \mathrm{z}$ (TOF ES+) $397.1\left([\mathrm{M}+\mathrm{Na}]^{+}, 100 \%\right) ; \mathrm{HRMS}$ $\mathrm{m} / \mathrm{z}$ (TOF ES+) $397.1991\left([\mathrm{M}+\mathrm{Na}]^{+} . \mathrm{C}_{21} \mathrm{H}_{34} \mathrm{NaO}_{2} \mathrm{Si}_{2}\right.$ requires 397.1995).

\section{$\left(3 S^{\star}, 4 R^{\star}, 6 S^{\star}, 7 E\right)$ 8-Phenyl-octa-1,7-diene-3,4,6-triol 9c}

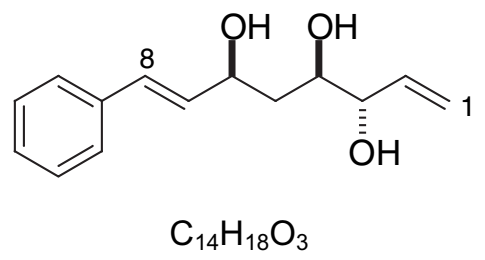

Exact Mass: 234.12560

Mol. Wt.: 234.29092

C, $71.77 ; \mathrm{H}, 7.74 ; \mathrm{O}, 20.49 \%$

$\mathrm{H}_{2} \mathrm{O}_{2}\left(1.130 \mathrm{~g}, 60 \%\right.$ in $\left.\mathrm{H}_{2} \mathrm{O}, 40.00 \mathrm{mmol}\right), \mathrm{KHCO}_{3}(600 \mathrm{mg}, 6.00 \mathrm{mmol})$ and $\mathrm{KF}(581 \mathrm{mg}, 10.00 \mathrm{mmol})$ were added to a solution of the crude products from the allylation of aldehyde $6 \mathbf{c}(1.244 \mathrm{~g}, 1.28 \mathrm{mmol}$ of oxasilinane 7c) in $\mathrm{MeOH}: \operatorname{THF}(1: 1,20 \mathrm{~mL})$ and the resulting mixture was stirred for 5 days. 
Aqueous work-up and purification by flash column chromatography (70 $\rightarrow 90 \%$ EtOAc in hexane) afforded trio/ $9 \mathrm{c}$ as a colorless viscous oil $(254 \mathrm{mg}, 85 \%) ; \mathrm{R}_{\mathrm{f}}=0.28$ (70\% EtOAc in hexane); $v_{\max }\left(\right.$ film) $/ \mathrm{cm}^{-1} 3367 \mathrm{vs}$ br (OH), 3082s, 3060s, 3026s, 2982m, 2916m, 1951w, 1878w, 1807w, 1646m $(\mathrm{C}=\mathrm{C}), 1599 \mathrm{~m}, 1578 \mathrm{~m}, 1494 \mathrm{~s}, 1449 \mathrm{~s}, 1426 \mathrm{~s}, 1315 \mathrm{~s}, 1183 \mathrm{~m}, 1110 \mathrm{~s}, 1070 \mathrm{~s}, 994 \mathrm{~s}, 969 \mathrm{~s}, 930 \mathrm{~s}, 857 \mathrm{~m}$, 750s, 694s; $\delta_{\mathrm{H}}(300 \mathrm{MHz}) 1.72-1.79\left(2 \mathrm{H}\right.$, stack, $\left.\mathrm{CH}_{2}\right), 2.50-3.70(3 \mathrm{H}, \mathrm{br}, 3 \times \mathrm{OH})$, 3.93-4.01 $(1 \mathrm{H}, \mathrm{m}, 4-$ $H), 4.16\left(1 \mathrm{H}\right.$, app. t, J 4.5, 3-H), $4.57\left(1 \mathrm{H}\right.$, app. q, J 6.6, 6- H), $5.26\left(1 \mathrm{H}, \mathrm{d}, J 10.7,=\mathrm{CH}_{c i s} \mathrm{H}_{\text {trans }}\right), 5.35$ $\left(1 \mathrm{H}, \mathrm{d}, J 17.3,=\mathrm{CH}_{\text {cis }} H_{\text {trans }}\right), 5.89\left(1 \mathrm{H}, \mathrm{ddd}, J 17.3,10.7,6.3, \mathrm{CH}=\mathrm{CH}_{2}\right), 6.21(1 \mathrm{H}, \mathrm{dd}, J 15.8,6.6$, $\mathrm{PhCH}=\mathrm{CH}), 6.60(1 \mathrm{H}, \mathrm{d}, J$ 15.8, $\mathrm{PhCH}=\mathrm{CH}), 7.20-7.40(5 \mathrm{H}$, stack, $\mathrm{PhH}) ; \delta_{\mathrm{C}}(75 \mathrm{MHz}) 37.6\left(\mathrm{CH}_{2}, \mathrm{C}-5\right)$, $72.8(\mathrm{CH}, 1 \times \mathrm{CHOH}), 74.2(\mathrm{CH}, 1 \times \mathrm{CHOH}), 75.7(\mathrm{CH}, 1 \times \mathrm{CHOH}), 117.5\left(\mathrm{CH}_{2},=\mathrm{CH}_{2}\right), 126.5(\mathrm{CH})$, $127.7(\mathrm{CH}), 128.6(\mathrm{CH}), 130.2(\mathrm{CH}), 131.5(\mathrm{CH}), 136.1(\mathrm{CH}), 136.4$ (quat. C, ipsoPh); $\mathrm{m} / \mathrm{z}$ (TOF ES+) $257.1\left([\mathrm{M}+\mathrm{Na}]^{+}, 100 \%\right) ; \mathrm{HRMS} \mathrm{m} / \mathrm{z}$ (TOF ES+) $257.1161\left([\mathrm{M}+\mathrm{Na}]^{+} . \mathrm{C}_{14} \mathrm{H}_{18} \mathrm{NaO}_{3}\right.$ requires 257.1154).

\section{Acetonide protection of triol 9c: 1-(2,2-dimethyl-5-vinyl-[1,3]dioxolan-4-yl)-4-phenyl-but-3-en-2-} ol 11c and 1-(2,2-dimethyl-6-styryl-[1,3]dioxan-4-yl)-prop-2-en-1-ol 12c

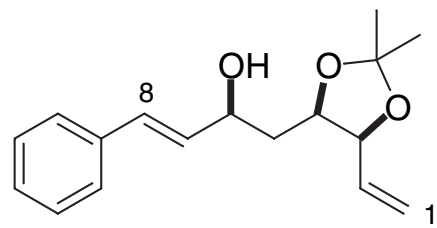

11c<smiles>CC1(C)OC(/C=C/c2ccccc2)C[C@H]([C@@H](O)C=[IH])O1</smiles>

12c

$$
\mathrm{C}_{17} \mathrm{H}_{22} \mathrm{O}_{3}
$$

Exact Mass: 274.15689

Mol. Wt.: 274.35478

C, $74.42 ; \mathrm{H}, 8.08 ; \mathrm{O}, 17.49 \%$

$\mathrm{Na}_{2} \mathrm{SO}_{4}(40 \mathrm{mg})$ and $p \mathrm{TsOH} \cdot \mathrm{H}_{2} \mathrm{O}(2 \mathrm{mg}, 16 \mu \mathrm{mol})$ were added to a solution of triol $9 \mathrm{c}$ (30 mg, 0.13 $\mathrm{mmol})$ in acetone $(1.3 \mathrm{~mL})$ and the resulting mixture was stirred overnight. Aqueous work-up afforded acetonides 11c and 12d as colorless oils and an inseparable mixture (73:27 11c:12c by ${ }^{1} \mathrm{H}$ NMR) (35 mg, quantitative mass recovery). ${ }^{1} \mathrm{H}-\mathrm{NMR}$ revealed the presence of other compounds that severely complicated spectral analysis. However, the two major acetonide products 11c and 12c still predominated and readily permitted assignment of the 1,2- and 1,3-relative stereochemistry of triol 9c); $\delta_{\mathrm{H}}\left(\mathrm{C}_{6} \mathrm{D}_{6}, 300 \mathrm{MHz}\right)$-identifiable resonances- $1.22\left(3 \mathrm{H}, \mathrm{s}, 1 \times \mathrm{CH}_{3}\right), 1.29\left(3 \mathrm{H}, \mathrm{s}, 1 \times \mathrm{CH}_{3}\right), 1.44(3 \mathrm{H}, \mathrm{s}, 1$ $\left.\times \mathrm{CH}_{3}\right), 1.51\left(3 \mathrm{H}, \mathrm{s}, 1 \times \mathrm{CH}_{3}\right), 5.55-5.70(1 \mathrm{H}, \mathrm{m}, 2-\mathrm{H} 11 \mathrm{c}), 5.71-5.86(1 \mathrm{H}, \mathrm{m}, 2-\mathrm{H} 12 \mathrm{c}) ; \delta_{\mathrm{H}}\left(\mathrm{C}_{6} \mathrm{D}_{6}, 75\right.$ $\mathrm{MHz})$-identifiable resonances- $19.9\left(\mathrm{CH}_{3}, 1 \times \mathrm{CH}_{3} 12 \mathrm{c}\right), 25.7\left(\mathrm{CH}_{3}, 1 \times \mathrm{CH}_{3} 11 \mathrm{c}\right), 28.2\left(\mathrm{CH}_{3}, 1 \times \mathrm{CH}_{3}\right.$ 11c), $30.3\left(\mathrm{CH}_{3}, 1 \times \mathrm{CH}_{3} 12 \mathrm{c}\right), 38.3\left(\mathrm{CH}_{2}, \mathrm{C}-5 \mathrm{12c}\right), 38.5\left(\mathrm{CH}_{2}, \mathrm{C}-511 \mathrm{c}\right), 71.8(\mathrm{CH}, 1 \times \mathrm{CH}(\mathrm{O}) 11 \mathrm{c})$, $77.5(\mathrm{CH}, 1 \times \mathrm{CH}(\mathrm{O}) 11 \mathrm{c}) 79.8(\mathrm{CH}, 1 \times \mathrm{CH}(\mathrm{O}) 11 \mathrm{c}), 98.8$ (quat. $\mathrm{C}, \mathrm{C}\left(\mathrm{CH}_{3}\right)_{2}$ 12c), 108.8 (quat. $\mathrm{C}$, $\mathrm{C}\left(\mathrm{CH}_{3}\right)_{2}$ 11c). 


\section{Allylation reaction of Aldehyde $6 \mathrm{~d}$ :}

TMSOTf (100 $\mu \mathrm{L}, 0.55 \mathrm{mmol})$ was added to a solution of aldehyde $6 \mathbf{d}(249 \mathrm{mg}, 0.55 \mathrm{mmol})$ and 2,6DTBMP (137 mg, $0.66 \mathrm{mmol})$ in $\mathrm{CH}_{2} \mathrm{Cl}_{2}(6 \mathrm{~mL})$ at $-78{ }^{\circ} \mathrm{C}$ and the reaction mixture was stirred for $16 \mathrm{~h}$. Aqueous work-up and removal of the solvent afforded oxasilinanes $\mathbf{7 d}$ and $\mathbf{8} \mathbf{d}$ (80:12; inseparable mixture) as a yellow liquid (385 mg, 92\%, remaining mass being diene products). This was used in the following oxidation step without any further purification. We were unable to separate the two diastereoisomers by preparative HPLC.
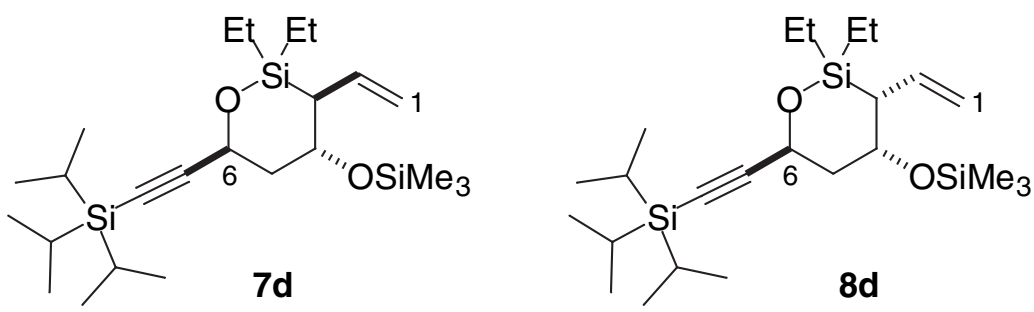

$$
\mathrm{C}_{24} \mathrm{H}_{48} \mathrm{O}_{2} \mathrm{Si}_{2}
$$

Exact Mass: 374.20973

Mol. Wt.: 374.66446

C, 67.32; H, 9.15; O, 8.54; Si, 14.99\%

$\left(3 S^{*}, 4 R^{\star}, 6 S^{\star}\right)$ 8-Triisopropylsilanyl-oct-1-en-7-yne-3,4,6-triol 9d<smiles>CC(C)[Si](C#CC(O)CC(O)[C@H](O)C=[IH])(C(C)C)C(C)C</smiles>

$$
\mathrm{C}_{17} \mathrm{H}_{32} \mathrm{O}_{3} \mathrm{Si}
$$

Exact Mass: 312.21207

Mol. Wt.: 312.51968

C, 65.33; H, 10.32; O, 15.36; Si, 8.99\%

$\mathrm{H}_{2} \mathrm{O}_{2}\left(623 \mathrm{mg}, 60 \%\right.$ in $\left.\mathrm{H}_{2} \mathrm{O}, 11.00 \mathrm{mmol}\right), \mathrm{KHCO}_{3}(132 \mathrm{mg}, 1.32 \mathrm{mmol})$ and $\mathrm{KF}(128 \mathrm{mg}, 2.20 \mathrm{mmol})$ were added to a solution of the crude products from the allylation of aldehyde $6 \mathbf{d}(385 \mathrm{mg}, 0.44 \mathrm{mmol}$ of oxasilinane $7 \mathrm{~d})$ in $\mathrm{MeOH}$ :THF $(1: 1,6 \mathrm{~mL})$ and the resulting mixture was stirred for 5 days. Aqueous work-up and purification by flash column chromatography ( $70 \rightarrow 90 \%$ EtOAc in hexane) afforded triol $9 \mathrm{~d}$ as a colorless viscous oil (47 mg, 34\%); $R_{\mathrm{f}}=0.30$ (50\% EtOAc in hexane); $v_{\max }(\mathrm{film}) / \mathrm{cm}^{-1} 3368 \mathrm{~s} \mathrm{br}$ (OH), 2944s, 2893s, 2868s, 2735m, 2171m (C $\equiv \mathrm{C}), 1723 \mathrm{w}, 1645 \mathrm{w}(\mathrm{C}=\mathrm{C}), 1464 \mathrm{~s}, 1427 \mathrm{~m}, 1384 \mathrm{~m}$, $1367 \mathrm{~m}, 1316 \mathrm{~m}, 1245 \mathrm{~m}, 1187 \mathrm{w}, 1061 \mathrm{~s}, 1018 \mathrm{~s}, 996 \mathrm{~s}, 925 \mathrm{~s}, 883 \mathrm{~s}, 849 \mathrm{~m}, 733 \mathrm{~m}, 678 \mathrm{~s} ; \delta_{\mathrm{H}}(300 \mathrm{MHz})$ 
$1.05\left(21 \mathrm{H}\right.$, br s, Si $\left.\left(\mathrm{CH}\left(\mathrm{CH}_{3}\right)_{2}\right)_{3}\right), 1.80-1.98\left(2 \mathrm{H}\right.$, stack, $\left.\mathrm{CH}_{2}\right), 3.15-3.49(3 \mathrm{H}, \mathrm{br}, 3 \times \mathrm{OH}), 3.89-3.98(1 \mathrm{H}$, $\mathrm{m}, 1 \times \mathrm{CHOH}), 4.12-4.20(1 \mathrm{H}, \mathrm{m}, 1 \times \mathrm{CHOH}), 4.64(1 \mathrm{H}, \mathrm{dd}, J 7.7,5.5,1 \times \mathrm{CHOH}), 5.25(1 \mathrm{H}, \mathrm{d}, J 10.8$, $\left.=\mathrm{CH}_{\text {cis }} \mathrm{H}_{\text {trans }}\right), 5.34\left(1 \mathrm{H}, \mathrm{d}, J 17.3,=\mathrm{CH}_{\text {cis }} H_{\text {trans }}\right), 5.86(1 \mathrm{H}, \mathrm{ddd}, J 17.3,10.8,6.1,2-H) ; \delta_{\mathrm{C}}(75 \mathrm{MHz}) 10.7$ $\left(\mathrm{CH}, \mathrm{Si}\left(\mathrm{CH}\left(\mathrm{CH}_{3}\right)_{2}\right)_{3}\right), 18.1\left(\mathrm{CH}_{3}, \mathrm{Si}\left(\mathrm{CH}\left(\mathrm{CH}_{3}\right)_{2}\right)_{3}\right), 38.7\left(\mathrm{CH}_{2}, \mathrm{C}-5\right), 61.8(\mathrm{CH}, 1 \times \mathrm{CHOH}), 73.2(\mathrm{CH}, 1 \times$ $\mathrm{CHOH}$ ), $75.6\left(\mathrm{CH}, 1 \times \mathrm{CHOH}\right.$ ), 86.1 (quat. $\mathrm{C}, 1 \times \mathrm{C} \equiv \mathrm{C}$ ), 108. 1 (quat. $\mathrm{C}, 1 \times \mathrm{C} \equiv \mathrm{C}$ ), $117.7\left(\mathrm{CH}_{2},=\mathrm{CH}_{2}\right.$ ), $136.4\left(\mathrm{CH}, \mathrm{CH}=\mathrm{CH}_{2}\right) ; \mathrm{m} / \mathrm{z}$ (TOF ES+) $335.1\left([\mathrm{M}+\mathrm{Na}]^{+}, 100 \%\right) ; \mathrm{HRMS} \mathrm{m} / \mathrm{z}$ (TOF ES+) 335.2020 $\left([\mathrm{M}+\mathrm{Na}]^{+} . \mathrm{C}_{17} \mathrm{H}_{32} \mathrm{NaO}_{3} \mathrm{Si}\right.$ requires 335.2018).

Acetonide protection of triol 9d (and trace 10d): 1-(2,2-dimethyl-5-vinyl-[1,3]dioxolan-4-yl)-4triisopropylsilanyl-but-3-yn-2-ol 11d and 1-\{2,2-dimethyl-6-[(triisopropylsilanyl)-ethynyl][1,3]dioxan-4-yl\}-prop-2-en-1-ol 12d and 1-(2,2-dimethyl-5-vinyl-[1,3]dioxolan-4-yl)-4triisopropylsilanyl-but-3-yn-2-ol 13d

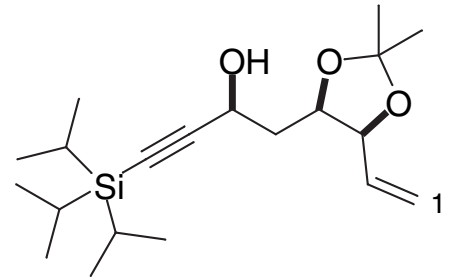

$11 d$

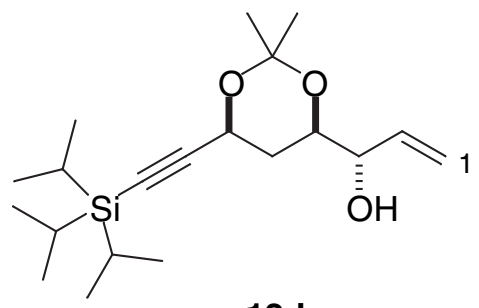

$12 d$

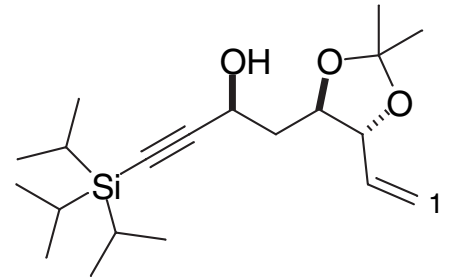

$13 d$

$$
\mathrm{C}_{20} \mathrm{H}_{36} \mathrm{O}_{3} \mathrm{Si}
$$

Exact Mass: 352.24337

Mol. Wt.: 352.58354

C, 68.13; H, 10.29; O, 13.61; Si, $7.97 \%$

$\mathrm{Na}_{2} \mathrm{SO}_{4}(40 \mathrm{mg})$ and $p \mathrm{TsOH} \cdot \mathrm{H}_{2} \mathrm{O}(2 \mathrm{mg}, 16 \mu \mathrm{mol})$ were added to a solution of trio/ $9 \mathbf{d}(30 \mathrm{mg}, 0.10$ $\mathrm{mmol})$ in acetone $(1.0 \mathrm{~mL})$ and the resulting mixture was stirred overnight. Aqueous work-up afforded acetonides $11 \mathbf{d}, \mathbf{1 2} \mathbf{d}$ and $\mathbf{1 3} \mathbf{d}$ as a colorless oil and an inseparable mixture ( $90 \% \mathbf{1 1 d}$ by $\left.{ }^{1} \mathrm{H} N M R\right)$ (35 $\mathrm{mg}$, quantitative); due to resonance overlaps and the small relative proportions of $\mathbf{1 2} \mathbf{d}$ and $\mathbf{1 3 d}$, only resonances for $11 \mathrm{~d}$ could be completely assigned in the ${ }^{1} \mathrm{H}$ NMR spectrum; $\delta_{\mathrm{H}}\left(\mathrm{C}_{6} \mathrm{D}_{6}, 300 \mathrm{MHz}\right.$ for 11d) $1.23\left(21 \mathrm{H}, \mathrm{s}, \mathrm{Si}\left(\mathrm{CH}\left(\mathrm{CH}_{3}\right)_{2}\right)_{3}\right), 1.37\left(3 \mathrm{H}, \mathrm{s}, 1 \times \mathrm{C}\left(\mathrm{CH}_{3}\right)_{2}\right), 1.51\left(3 \mathrm{H}, \mathrm{s}, 1 \times \mathrm{C}\left(\mathrm{CH}_{3}\right)_{2}\right), 1.80(1 \mathrm{H}$, ddd, J 13.7, 7.8, 2.6, 5- $\left.\mathrm{H}_{\mathrm{a}} \mathrm{H}_{\mathrm{b}}\right), 2.02-2.17\left(1 \mathrm{H}, \mathrm{m}, 5-\mathrm{H}_{\mathrm{a}} \mathrm{H}_{\mathrm{b}}\right), 2.42-2.70(1 \mathrm{H}, \mathrm{br}, \mathrm{OH}), 4.42-4.53(2 \mathrm{H}$, stack, 3- $\mathrm{H}$, 4-H), $4.73(1 \mathrm{H}, \mathrm{dd}, J 7.8,6.6,6-H), 5.05\left(1 \mathrm{H}, \mathrm{dd}, J 10.4,1.3,1-H_{\text {cis }}\right), 5.21\left(1 \mathrm{H}, \mathrm{d}, J 16.9,1-H_{\text {trans }}\right), 5.70$ $(1 \mathrm{H}$, ddd, $J 16.9,10.3,2.6,2-H) ; \delta_{C}\left(C_{6} D_{6}, 75 \mathrm{MHz}\right.$; those resonances that are not specifically assigned belong to either 12d or $13 \mathbf{d}) 10.6\left(\mathrm{CH}, \mathrm{Si}\left(\mathrm{CH}\left(\mathrm{CH}_{3}\right)_{2}\right)_{3}\right), 10.8\left(\mathrm{CH}, \mathrm{Si}\left(\mathrm{CH}\left(\mathrm{CH}_{3}\right)_{2}\right)_{3} \mathbf{1 1 d}\right), 11.2(\mathrm{CH}$, $\left.\mathrm{Si}\left(\mathrm{CH}\left(\mathrm{CH}_{3}\right)_{2}\right)_{3}\right), 18.21 \quad\left(\mathrm{CH}_{3}, \quad \mathrm{Si}\left(\mathrm{CH}\left(\mathrm{CH}_{3}\right)_{2}\right)_{3}\right), 18.24\left(\mathrm{CH}_{3}, \mathrm{Si}\left(\mathrm{CH}\left(\mathrm{CH}_{3}\right)_{2}\right)_{3} \quad 11 d\right), 18.4\left(\mathrm{CH}_{3}\right.$, $\left.\mathrm{Si}\left(\mathrm{CH}\left(\mathrm{CH}_{3}\right)_{2}\right)_{3}\right), 18.8\left(\mathrm{CH}_{3},\left(\mathrm{CH}_{3}\right)_{\text {pseudoax. }}\right.$ 12d $), 25.0\left(\mathrm{CH}_{3},\left(\mathrm{CH}_{3}\right)_{\text {pseudoax. }}\right.$ 11d $), 26.5\left(\mathrm{CH}_{3}, 1 \times \mathrm{CH}_{3} 13 \mathrm{~d}\right)$, $26.8\left(\mathrm{CH}_{3}, 1 \times \mathrm{CH}_{3}\right.$ 13d $), 27.7\left(\mathrm{CH}_{3},\left(\mathrm{CH}_{3}\right)_{\text {pseudoeq. }} 11 \mathrm{~d}\right), 29.5\left(\mathrm{CH}_{3},\left(\mathrm{CH}_{3}\right)_{\text {pseudoeq. }} 12 \mathrm{~d}\right), 38.9\left(\mathrm{CH}_{2}, \mathrm{C}-5\right.$ 11d), $39.8\left(\mathrm{CH}_{2}, 1 \times \mathrm{C}-5\right), 60.6(\mathrm{CH}, 1 \times \mathrm{CH}), 61.0(\mathrm{CH}, 1 \times \mathrm{CH}), 61.4(\mathrm{CH}, 1 \times \mathrm{CH} 11 \mathrm{~d}), 71.7(\mathrm{CH}, 1 \times$ 
$\mathrm{CH}), 73.9(\mathrm{CH}, 1 \times \mathrm{CH}), 76.1(\mathrm{CH}, 1 \times \mathrm{CH} 11 \mathrm{~d}), 78.5(\mathrm{CH}, 1 \times \mathrm{CH}), 79.5(\mathrm{CH}, 1 \times \mathrm{CH} 11 \mathrm{~d}), 82.5(\mathrm{CH}$, $1 \times \mathrm{CH}$ ), 84.8 (quat. $\mathrm{C}, 1 \times \mathrm{SiC} \equiv \mathrm{C}$ ), 85.1 (quat. $\mathrm{C}, 1 \times \mathrm{SiC} \equiv \mathrm{C} 11 \mathrm{~d}$ ), 85.2 (quat. $\mathrm{C}, 1 \times \mathrm{SiC} \equiv \mathrm{C}$ ), 99.1 (quat. $\mathrm{C}, \mathrm{C}\left(\mathrm{CH}_{3}\right)_{2}$ 12d), 107.5 (quat. $\mathrm{C}, \mathrm{C}\left(\mathrm{CH}_{3}\right)_{2}$ 13d), 108.7 (quat. $\mathrm{C}, \mathrm{C}\left(\mathrm{CH}_{3}\right)_{2}$ 11d), 109.1 (quat. $\mathrm{C}, 1$ $\times \mathrm{SiC} \equiv \mathrm{C}$ ), 109.5 (quat. $\mathrm{C}, 1 \times \mathrm{SiC} \equiv \mathrm{C}$ ), 109.6 (quat. $\mathrm{C}, 1 \times \mathrm{SiC} \equiv \mathrm{C} 11 \mathrm{~d}$ ), $116.2\left(\mathrm{CH}_{2}, 1 \times \mathrm{C}-1\right), 117.7$ $\left(\mathrm{CH}_{2}, \mathrm{C}-111 \mathrm{~d}\right), 118.1\left(\mathrm{CH}_{2}, 1 \times \mathrm{C}-1\right), 135.0(\mathrm{CH}, \mathrm{C}-211 \mathrm{~d}), 135.7(\mathrm{CH}, 1 \times \mathrm{C}-2), 136.6(\mathrm{CH}, 1 \times \mathrm{C}-2)$.

\section{Allylation reaction of Aldehyde 6e:}

TMSOTf $(324 \mu \mathrm{L}, 1.8 \mathrm{mmol})$ was added to a solution of aldehyde $6 \mathrm{e}(607 \mathrm{mg}, 1.8 \mathrm{mmol})$ and 2,6DTBMP (443 mg, $2.2 \mathrm{mmol})$ in $\mathrm{CH}_{2} \mathrm{Cl}_{2}(18 \mathrm{~mL})$ at $-78{ }^{\circ} \mathrm{C}$ and the reaction mixture was stirred for $8 \mathrm{~h}$. Aqueous work-up and removal of the solvent afforded compounds $7 \mathbf{e}$ and $8 \mathbf{e}$ (69:14; inseparable mixture) as a yellow liquid ( $1.003 \mathrm{~g}, 83 \%$, remaining mass being diene products). This was used in the following step without any further purification. Samples of compounds $7 \mathbf{e}$ and $8 \mathbf{e}$ were obtained as colorless oils by preparative HPLC $\left(5 \% \mathrm{H}_{2} \mathrm{O}\right.$ in $\left.\mathrm{MeCN}\right) ; 7$ e $t_{\mathrm{R}}=74.9 \mathrm{~min}$ (contaminated with residual diene $5 \mathbf{e}$ ) and $8 \mathbf{e} t_{\mathrm{R}}=85.2 \mathrm{~min}$.

$\left(3 S^{\star}, 4 R^{\star}, 6 S^{\star}\right)$ oxasilinane $7 \mathrm{e}$

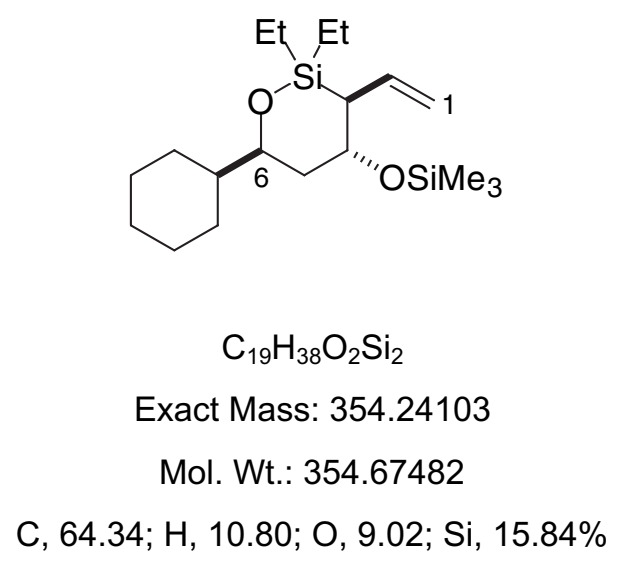

HPLC: $t_{R}=74.9 \mathrm{~min} ; v_{\max }($ film $) / \mathrm{cm}^{-1} 3075 \mathrm{w}, 2954 \mathrm{~s}, 2926 \mathrm{~s}, 2876 \mathrm{~s}, 2853 \mathrm{~s}, 1626 \mathrm{~m}(\mathrm{C}=\mathrm{C}), 1450 \mathrm{~m}$, $1414 \mathrm{~m}, 1378 \mathrm{w}, 1347 \mathrm{w}, 1306 \mathrm{w}, 1251 \mathrm{~s}, 1152 \mathrm{~m}, 1002 \mathrm{~s}, 969 \mathrm{~m}, 939 \mathrm{~m}, 896 \mathrm{~m}, 872 \mathrm{~m}, 841 \mathrm{~s}, 810 \mathrm{w}, 749 \mathrm{~m}$, $724 \mathrm{~m}, 685 \mathrm{w} ; \delta_{\mathrm{H}}(300 \mathrm{MHz}) 0.10\left(9 \mathrm{H}, \mathrm{s}, \mathrm{Si}\left(\mathrm{CH}_{3}\right)_{3}\right), 0.44-0.86\left(4 \mathrm{H}\right.$, stack, OSi $\left.\left(\mathrm{CH}_{2} \mathrm{CH}_{3}\right)_{2}\right), 0.85-1.42$ $\left(12 \mathrm{H}\right.$, stack including [0.92 $\left.\left(3 \mathrm{H}, \mathrm{t}, J 7.7, \mathrm{OSi}\left(\mathrm{CH}_{2} \mathrm{CH}_{3}\right)\right), 0.97\left(3 \mathrm{H}, \mathrm{t}, J 7.7, \mathrm{OSi}\left(\mathrm{CH}_{2} \mathrm{CH}_{3}\right)\right)\right]$, $\mathrm{OSi}\left(\mathrm{CH}_{2} \mathrm{CH}_{3}\right)_{2}, 6 \times$ cyclohexylH), 1.51-2.01 (8H, stack, 5- $\mathrm{CH}_{2}, 3-\mathrm{H}, 5 \times$ cyclohexyl $\left.H\right), 3.78(1 \mathrm{H}$, app. td, J 7.7, 3.1, CH(OSi)), 4.07 (1H, app. td, J 6.6, 2.2, CH(OSi)), $4.90\left(1 \mathrm{H}, \mathrm{d}, J 11.4,1-H_{c i s}\right), 4.91(1 \mathrm{H}, \mathrm{d}, J$ 15.8, 1- $\left.H_{\text {trans }}\right), 5.67-5.83(1 \mathrm{H}, \mathrm{m}, 2-\mathrm{H}) ; \delta_{\mathrm{C}}(75 \mathrm{MHz}) 0.2\left(\mathrm{CH}_{3}, \mathrm{Si}\left(\mathrm{CH}_{3}\right)_{3}\right), 5.2\left(\mathrm{CH}_{2}, \mathrm{Si}\left(\mathrm{CH}_{2} \mathrm{CH}_{3}\right)\right), 5.5$ $\left.\left(\mathrm{CH}_{2}, \mathrm{Si}\left(\mathrm{CH}_{2} \mathrm{CH}_{3}\right)\right), 6.3\left(\mathrm{CH}_{3}, \mathrm{Si}\left(\mathrm{CH}_{2} \mathrm{CH}_{3}\right)\right), 6.6\left(\mathrm{CH}_{3}, \mathrm{Si}\left(\mathrm{CH}_{2} \mathrm{CH}_{3}\right)\right), 26.1\left(\mathrm{CH}_{2} \text {, cyclohexylCH}\right)_{2}\right), 26.3$ $\left.\left(\mathrm{CH}_{2} \text {, cyclohexylCH}\right)_{2}, 26.6\left(\mathrm{CH}_{2} \text {, cyclohexylCH}\right)_{2}, 29.1\left(\mathrm{CH}_{2}, 2 \times \text { cyclohexylCH}\right)_{2}\right), 36.7\left(\mathrm{CH}_{2}, \mathrm{C}-5\right)$, $39.7(\mathrm{CH}, \mathrm{C}-3), 43.8(\mathrm{CH}, \mathrm{C}-7), 71.0(\mathrm{CH}, \mathrm{CH}(\mathrm{OSi})), 73.3(\mathrm{CH}, \mathrm{CH}(\mathrm{OSi})), 113.4\left(\mathrm{CH}_{2}, \mathrm{C}-1\right), 137.6(\mathrm{CH}$, $\mathrm{C}-2) ; \mathrm{m} / \mathrm{z}$ (TOF ES+) $377.3\left([\mathrm{M}+\mathrm{Na}]^{+}, 100 \%\right) ; \mathrm{HRMS} \mathrm{m} / \mathrm{z}$ (TOF ES+) $377.2312\left([\mathrm{M}+\mathrm{Na}]^{+}\right.$. $\mathrm{C}_{19} \mathrm{H}_{38} \mathrm{NaO}_{2} \mathrm{Si}_{2}$ requires 377.2308). 
<smiles>C=C[C@H]1[C@@H](OC)C[C@@H](C2CCCCC2)O[Si]1(CC)CC</smiles>

$$
\mathrm{C}_{19} \mathrm{H}_{38} \mathrm{O}_{2} \mathrm{Si}_{2}
$$

Exact Mass: 354.24103

Mol. Wt.: 354.67482

C, $64.34 ; \mathrm{H}, 10.80 ; \mathrm{O}, 9.02 ; \mathrm{Si}, 15.84 \%$

HPLC: $t_{\mathrm{R}}=85.2 \mathrm{~min} ; v_{\max }(\mathrm{film}) / \mathrm{cm}^{-1} 3075 \mathrm{w}, 2955 \mathrm{~s}, 2927 \mathrm{~s}, 2875 \mathrm{~m}, 2854 \mathrm{~m}, 1626 \mathrm{w}, 1451 \mathrm{~m}, 1416 \mathrm{w}$, $1378 w, 1349 w, 1325 w, 1302 w, 1273 w, 1251 \mathrm{~m}, 1205 w, 1170 w, 1132 \mathrm{~m}, 1103 \mathrm{~m}, 1082 \mathrm{~m}, 1058 \mathrm{~m}$, $1047 \mathrm{~m}, 1003 \mathrm{~m}, 963 \mathrm{~m}, 921 \mathrm{w}, 898 \mathrm{~m}, 865 \mathrm{~m}, 841 \mathrm{~s}, 815 \mathrm{~m}, 794 \mathrm{w}, 772 \mathrm{w}, 722 \mathrm{~m}, 665 \mathrm{~m} ; \delta_{\mathrm{H}}(400 \mathrm{MHz}) 0.10$ $\left(9 \mathrm{H}, \mathrm{s}, \mathrm{Si}\left(\mathrm{CH}_{3}\right)_{3}\right), 0.47-0.86\left(4 \mathrm{H}\right.$, stack, OSi $\left.\left(\mathrm{CH}_{2} \mathrm{CH}_{3}\right)_{2}\right), 0.91-1.42(13 \mathrm{H}$, stack including $[0.94(3 \mathrm{H}, \mathrm{t}, \mathrm{J}$ 7.9, OSi $\left.\left(\mathrm{CH}_{2} \mathrm{CH}_{3}\right)\right), 0.97\left(3 \mathrm{H}, \mathrm{t}, J\right.$ 7.9, OSi $\left.\left(\mathrm{CH}_{2} \mathrm{CH}_{3}\right)\right), 1.35-1.41\left(1 \mathrm{H}, \mathrm{m}, 5-\mathrm{H}_{\text {pseudoax. }}\right)$ ], OSi $\left(\mathrm{CH}_{2} \mathrm{CH}_{3}\right)_{2}, 5-$ $H_{\text {pseudoax. }}, 6 \times$ cyclohexyl $\left.H\right), 1.61-1.85\left(7 \mathrm{H}\right.$, stack including [1.83 $\left(1 \mathrm{H}, \mathrm{dd}, J\right.$ 9.9, 2.6, 3-H)], 5- $H_{\text {pseudoeq. }}$, 3-H, $5 \times$ cyclohexylH), $3.91(1 \mathrm{H}, \mathrm{dd}, J 10.2,5.6,6-H), 4.22-4.28(1 \mathrm{H}, \mathrm{m}, 4-H), 4.84(1 \mathrm{H}, \mathrm{d}, J 10.4,1-$ $\left.H_{\text {cis }}\right), 4.87\left(1 \mathrm{H}, \mathrm{d}, J 17.5,1-H_{\text {trans }}\right), 5.87\left(1 \mathrm{H}\right.$, app. dt, J 17.5, 10.2, 2-H); $\delta_{\mathrm{C}}(100 \mathrm{MHz}) 0.2\left(\mathrm{CH}_{3}\right.$, $\left.\mathrm{Si}\left(\mathrm{CH}_{3}\right)_{3}\right), 4.8\left(\mathrm{CH}_{2}, \mathrm{OSi}\left(\mathrm{CH}_{2} \mathrm{CH}_{3}\right)\right), 5.4\left(\mathrm{CH}_{2}, \mathrm{OSi}\left(\mathrm{CH}_{2} \mathrm{CH}_{3}\right)\right), 6.60\left(\mathrm{CH}_{3}, \mathrm{OSi}\left(\mathrm{CH}_{2} \mathrm{CH}_{3}\right)\right), 6.63\left(\mathrm{CH}_{3}\right.$, OSi $\left.\left(\mathrm{CH}_{2} \mathrm{CH}_{3}\right)\right)$, [26.4, 26.8, 28.5, $28.7\left(\mathrm{CH}_{2}\right.$, cyclohexylCH , some overlap), $38.7\left(\mathrm{CH}_{2}, \mathrm{C}-5\right), 38.8(\mathrm{CH}$, C-3), $44.3(\mathrm{CH}, \mathrm{C}-7), 70.9(\mathrm{CH}, \mathrm{C}-6), 71.6(\mathrm{CH}, \mathrm{C}-4), 112.3\left(\mathrm{CH}_{2}, \mathrm{C}-1\right), 138.4(\mathrm{CH}, \mathrm{C}-2) ; \mathrm{m} / \mathrm{z}$ (TOF ES+) $377.3\left([\mathrm{M}+\mathrm{Na}]^{+}, 100 \%\right)$; HRMS $\mathrm{m} / \mathrm{z}$ (TOF ES+) $377.2299\left([\mathrm{M}+\mathrm{Na}]^{+} . \mathrm{C}_{19} \mathrm{H}_{38} \mathrm{NaO}_{2} \mathrm{Si}_{2}\right.$ requires 377.2308).

$\left(1 S^{\star}, 3 R^{\star}, 4 S^{\star}\right)$ 1-Cyclohexyl-hex-5-ene-1,3,4-triol 9e<smiles>OC(C=[IH])C(O)CC(O)C1CCCCC1</smiles>

\author{
$\mathrm{C}_{12} \mathrm{H}_{22} \mathrm{O}_{3}$ \\ Exact Mass: 214.15689 \\ Mol. Wt.: 214.30128 \\ C, $67.26 ; \mathrm{H}, 10.35 ; \mathrm{O}, 22.40 \%$
}


$\mathrm{H}_{2} \mathrm{O}_{2}\left(1.133 \mathrm{~g}, 60 \%\right.$ in $\left.\mathrm{H}_{2} \mathrm{O}, 20.00 \mathrm{mmol}\right), \mathrm{KHCO}_{3}$ (300 mg, $\left.3.00 \mathrm{mmol}\right)$ and $\mathrm{KF}(291 \mathrm{mg}, 5.00 \mathrm{mmol})$ were added to a solution of the products from the allylation of aldehyde $6 \mathrm{e}(663 \mathrm{mg}, 0.72 \mathrm{mmol}$ of oxasilinane 7e) in MeOH:THF $(1: 1,10 \mathrm{~mL})$ and the resulting mixture was stirred for 5 days. Aqueous work-up and purification by flash column chromatography ( $50 \rightarrow 70 \%$ EtOAc in hexane) afforded triol $9 \mathrm{e}$ as a colorless viscous oil (78 mg, 51\%); $\mathrm{R}_{\mathrm{f}}=0.30$ (50\% EtOAc in hexane); $v_{\max }(\mathrm{film}) / \mathrm{cm}^{-1} 3368 \mathrm{br} \mathrm{s}$ $(\mathrm{OH}), 2926 \mathrm{~s}, 2852 \mathrm{~s}, 1645 \mathrm{w}(\mathrm{C}=\mathrm{C}), 1450 \mathrm{~s}, 1318 \mathrm{~m}, 1180 \mathrm{~m}, 1065 \mathrm{~m}, 994 \mathrm{~m}, 926 \mathrm{~m}, 893 \mathrm{~m}, 847 \mathrm{~m} ; \delta_{\mathrm{H}}(300$ $\mathrm{MHz})$ 0.80-1.84 (13H, stack, 3- $\mathrm{CH}_{2}, 11 \times$ cyclohexylH), 2.90-3.78 $(4 \mathrm{H}$, stack including [3.58-3.67 $(1 \mathrm{H}$, $\mathrm{m}, 1 \times \mathrm{CHOH})$ ], $1 \times \mathrm{CHOH}, 3 \times \mathrm{OH}), 3.88(1 \mathrm{H}$, app. dt, J 9.7, 3.1, $1 \times \mathrm{CHOH}), 4.12(1 \mathrm{H}$, app. t, J 4.6, 1 $\times \mathrm{CHOH}), 5.23\left(1 \mathrm{H}, \mathrm{d}, J\right.$ 10.7, $\left.=\mathrm{CH}_{\text {cis }} \mathrm{H}_{\text {trans }}\right), 5.32\left(1 \mathrm{H}, \mathrm{d}, J 17.2,=\mathrm{CH}_{\text {cis }} H_{\text {trans }}\right), 5.87(1 \mathrm{H}, \mathrm{ddd}, J 17.2$, 10.7, 6.3, $\left.\mathrm{CH}=\mathrm{CH}_{2}\right) ; \delta_{\mathrm{C}}(75 \mathrm{MHz}) 26.1\left(\mathrm{CH}_{2}\right), 26.2\left(\mathrm{CH}_{2}\right), 26.5\left(\mathrm{CH}_{2}\right), 27.8\left(\mathrm{CH}_{2}\right), 28.8\left(\mathrm{CH}_{2}\right), 33.7$ $\left(\mathrm{CH}_{2}, \mathrm{C}-2\right), 44.4(\mathrm{CH}$, cyclohexylCH$), 75.2(\mathrm{CH}, 1 \times \mathrm{CHOH}), 75.7(\mathrm{CH}, 1 \times \mathrm{CHOH}), 76.8(\mathrm{CH}, 1 \times$ $\mathrm{CHOH}), 117.2\left(\mathrm{CH}_{2},=\mathrm{CH}_{2}\right), 136.3\left(\mathrm{CH}, \mathrm{CH}=\mathrm{CH}_{2}\right) ; \mathrm{m} / \mathrm{z}$ (TOF ES+) $237.2\left([\mathrm{M}+\mathrm{Na}]^{+}, 100 \%\right) . \mathrm{HRMS} \mathrm{m} / \mathrm{z}$ (TOF ES+) $237.1460\left([\mathrm{M}+\mathrm{Na}]^{+} . \mathrm{C}_{12} \mathrm{H}_{22} \mathrm{NaO}_{3}\right.$ requires 237.1467).

Acetonide protection of Triol 9e: 1-cyclohexyl-2-(2,2-dimethyl-5-vinyl-[1,3]dioxolan-4-yl)-ethanol 11e and 1-(6-cyclohexyl-2,2-dimethyl-[1,3]dioxan-4-yl)-prop-2-en-1-ol 12e

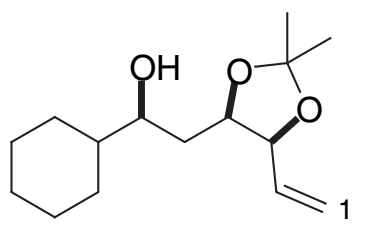

$11 \mathrm{e}$<smiles>CC1(C)OC(C2CCCCC2)CC([C@H](O)C=[IH])O1</smiles>

$12 e$

$$
\mathrm{C}_{15} \mathrm{H}_{26} \mathrm{O}_{3}
$$

Exact Mass: 254.18820

Mol. Wt.: 254.36514

C, $70.83 ; \mathrm{H}, 10.30 ; \mathrm{O}, 18.87 \%$

$\mathrm{Na}_{2} \mathrm{SO}_{4}(40 \mathrm{mg})$ and $p \mathrm{TsOH} \cdot \mathrm{H}_{2} \mathrm{O}(2 \mathrm{mg}, 16 \mu \mathrm{mol})$ were added to a solution of triol $9 \mathrm{e}(30 \mathrm{mg}, 0.14$ $\mathrm{mmol})$ in acetone $(1.4 \mathrm{~mL}$ ) and the resulting mixture was stirred overnight. Aqueous work-up afforded acetonides $11 \mathrm{e}$ and $12 \mathrm{e}$ as a colorless oil and an inseparable mixture (40:60 11e:12e by ${ }^{1} \mathrm{H}$ NMR) (36 $\mathrm{mg}$, quantitative); Data reported on the mixture of $11 \mathrm{e}$ and $12 \mathrm{e}: \delta_{\mathrm{H}}(300 \mathrm{MHz}) 0.80-2.09(38 \mathrm{H}$, stack including [1.21 (3H, s, $\left.\left.1 \times \mathrm{CH}_{3}\right), 1.26\left(3 \mathrm{H}, \mathrm{s}, 1 \times \mathrm{CH}_{3}\right), 1.40\left(3 \mathrm{H}, \mathrm{s}, 1 \times \mathrm{CH}_{3}\right), 1.46\left(3 \mathrm{H}, \mathrm{s}, 1 \times \mathrm{CH}_{3}\right)\right], 4 \times$ $\mathrm{CH}_{3}, 22 \times$ cyclohexylH, $\left.2 \times 5-\mathrm{H}_{2}\right), 3.27-3.37(1 \mathrm{H}, \mathrm{m}), 3.51-3.59(1 \mathrm{H}, \mathrm{m}), 3.60-3.70(1 \mathrm{H}, \mathrm{m}), 4.00-4.09$ $(1 \mathrm{H}, \mathrm{m}), 4.13-4.21(1 \mathrm{H}, \mathrm{m}), 4.29(1 \mathrm{H}, \mathrm{t}, J 6.4), 5.01\left(1 \mathrm{H}, \mathrm{d}, J 10.3,1-H_{c i s} 11 \mathrm{e}\right), 5.13(1 \mathrm{H}, \mathrm{d}, J 10.3,1-$ $\left.H_{\text {cis }} 12 \mathrm{e}\right), 5.15\left(1 \mathrm{H}, \mathrm{d}, J 17.5,1-H_{\text {trans }} 11 \mathrm{e}\right), 5.44\left(1 \mathrm{H}, \mathrm{d}, J 16.8,1-H_{\text {trans }} 12 \mathrm{e}\right), 5.66(1 \mathrm{H}, \mathrm{ddd}, J 17.5$, 10.3, 7.0, 2-H 11e), $5.84\left(1 \mathrm{H}\right.$, ddd, $J$ 16.8, 10.3, 5.0, 2-H 12e), OH resonances not observed; $\delta_{\mathrm{C}}(100$ $\mathrm{MHz}) 19.9\left(\mathrm{CH}_{3}, 1 \times \mathrm{CH}_{3} 12 \mathrm{e}\right), 25.6\left(\mathrm{CH}_{3}, 1 \times \mathrm{CH}_{3} 11 \mathrm{e}\right), 26.4\left(\mathrm{CH}_{2}\right), 26.5\left(\mathrm{CH}_{2}\right), 26.7\left(\mathrm{CH}_{2}\right), 26.8$ $\left(\mathrm{CH}_{2}\right), 27.01\left(\mathrm{CH}_{2}\right), 27.8\left(\mathrm{CH}_{2}\right), 28.1\left(\mathrm{CH}_{3}, 1 \times \mathrm{CH}_{3} 11 \mathrm{e}\right), 28.2\left(\mathrm{CH}_{2}\right), 28.4\left(\mathrm{CH}_{2}\right), 28.9\left(\mathrm{CH}_{2}\right), 29.5$ 
$\left(\mathrm{CH}_{2}\right), 30.2\left(\mathrm{CH}_{2}\right), 30.3\left(\mathrm{CH}_{3}, 1 \times \mathrm{CH}_{3} 12 \mathrm{e}\right), 34.8\left(\mathrm{CH}_{2}\right), 43.3(\mathrm{CH}, 1 \times$ cyclohexylCH$), 44.4(\mathrm{CH}, 1 \times$ cyclohexylCH), $72.5(\mathrm{CH}, 1 \times \mathrm{CH}(\mathrm{O})), 73.0(\mathrm{CH}, 1 \times \mathrm{CH}(\mathrm{O})), 74.5(\mathrm{CH}, 1 \times \mathrm{CH}(\mathrm{O})), 75.5(\mathrm{CH}, 1 \times$ $\mathrm{CH}(\mathrm{O})), 79.1(\mathrm{CH}, 1 \times \mathrm{CH}(\mathrm{O})), 80.0(\mathrm{CH}, 1 \times \mathrm{CH}(\mathrm{O})), 98.5$ (quat. $\mathrm{C}, \mathrm{C}\left(\mathrm{CH}_{3}\right)_{2}$ 12e), 108.4 (quat. $\mathrm{C}$, $\left.\mathrm{C}\left(\mathrm{CH}_{3}\right)_{2} 11 \mathrm{e}\right), 115.8\left(\mathrm{CH}_{2}, 1 \times \mathrm{C}-1\right), 117.6\left(\mathrm{CH}_{2}, 1 \times \mathrm{C}-1\right), 134.9(\mathrm{CH}, 1 \times \mathrm{C}-2), 136.9(\mathrm{CH}, 1 \times \mathrm{C}-2)$.

\title{
Allylation reaction of Aldehyde 6f:
}

TMSOTf $(202 \mu \mathrm{L}, 1.12 \mathrm{mmol})$ was added to a solution of aldehyde $6 \mathrm{f}(369 \mathrm{mg}, 1.12 \mathrm{mmol})$ and 2,6DTBMP $(277 \mathrm{mg}, 1.35 \mathrm{mmol})$ in $\mathrm{CH}_{2} \mathrm{Cl}_{2}(12 \mathrm{~mL})$ at $-78{ }^{\circ} \mathrm{C}$ and the reaction mixture was stirred for $8 \mathrm{~h}$. Aqueous work-up and removal of the solvent afforded compounds $\mathbf{7 f}$ and $\mathbf{8 f}$ (68:8; inseparable mixture) as a colorless liquid ( $630 \mathrm{mg}, 76 \%$, remaining mass being diene products). This was used in the following step without any further purification. Analytically pure samples of each compound were obtained as colorless oils by preparative HPLC ( $\mathrm{t}=0 \rightarrow 40 \mathrm{~min}, 50 \rightarrow 100 \% \mathrm{MeCN}$ in $\mathrm{H}_{2} \mathrm{O}$ ); $7 \mathrm{f} t_{\mathrm{R}}=$ $66.9 \mathrm{~min}$ and $8 \mathrm{f} t_{\mathrm{R}}=69.0 \mathrm{~min}$.

$\left(3 S^{*}, 4 R^{*}, 6 R^{*}\right)$ oxasilinane $7 f$

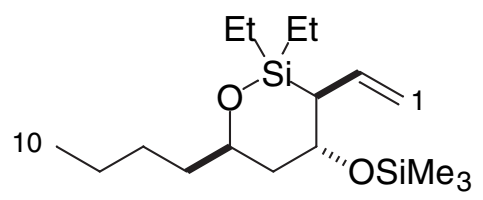

\author{
$\mathrm{C}_{17} \mathrm{H}_{36} \mathrm{O}_{2} \mathrm{Si}_{2}$ \\ Exact Mass: 328.22538 \\ Mol. Wt.: 328.63754 \\ C, $62.13 ; \mathrm{H}, 11.04 ; \mathrm{O}, 9.74 ; \mathrm{Si}, 17.09 \%$
}

HPLC: $t_{\mathrm{R}}=66.9 \mathrm{~min} ; v_{\max }($ film $) / \mathrm{cm}^{-1} 2957 \mathrm{~s}, 2876 \mathrm{~s}, 1628 \mathrm{~m}(\mathrm{C}=\mathrm{C}), 1462 \mathrm{~m}, 1378 \mathrm{w}, 1252 \mathrm{~s}, 1152 \mathrm{~m}$, $1115 \mathrm{~m}, 1067 \mathrm{~s}, 998 \mathrm{~m}, 946 \mathrm{w}, 871 \mathrm{w}, 841 \mathrm{~s}, 748 \mathrm{w}, 725 \mathrm{~m} ; \delta_{\mathrm{H}}(400 \mathrm{MHz}) 0.09\left(9 \mathrm{H}, \mathrm{s}, \mathrm{Si}\left(\mathrm{CH}_{3}\right)_{3}\right), 0.58(2 \mathrm{H}$, q, J 7.8, OSi $\left.\left(\mathrm{CH}_{2} \mathrm{CH}_{3}\right)\right), 0.65-0.80\left(2 \mathrm{H}, \mathrm{m}, \mathrm{OSi}\left(\mathrm{CH}_{2} \mathrm{CH}_{3}\right)\right), 0.90\left(3 \mathrm{H}, \mathrm{t}, J 7.0,10-\mathrm{CH}_{3}\right), 0.91(3 \mathrm{H}, \mathrm{t}, J$ 7.9, $\left.\mathrm{OSi}\left(\mathrm{CH}_{2} \mathrm{CH}_{3}\right)\right), 0.96\left(3 \mathrm{H}, \mathrm{t}, \mathrm{J}\right.$ 7.8, OSi $\left.\left(\mathrm{CH}_{2} \mathrm{CH}_{3}\right)\right), 1.23-1.45\left(5 \mathrm{H}\right.$, stack, 9- $\left.\mathrm{CH}_{2}, 8-\mathrm{CH}_{2}, 7-\mathrm{CH}_{\mathrm{a}} \mathrm{H}_{\mathrm{b}}\right), 1.49-$ $1.56\left(1 \mathrm{H}, \mathrm{m}, 7-\mathrm{CH}_{\mathrm{a}} \mathrm{H}_{b}\right), 1.61\left(1 \mathrm{H}\right.$, ddd, J 14.1, 7.6, 3.7, 5- $\left.\mathrm{CH}_{\mathrm{a}} \mathrm{H}_{\mathrm{b}}\right), 1.70(1 \mathrm{H}$, ddd, J 14.1, 6.8, 2.8, 5$\left.\mathrm{CH}_{\mathrm{a}} \mathrm{H}_{b}\right), 1.88(1 \mathrm{H}, \mathrm{dd}, \mathrm{J} 10.4,6.9,3-H), 4.03-4.11\left(2 \mathrm{H}\right.$, stack, 4-H, 6-H), 4.88-4.94 (2H, stack, 1-CH $\mathrm{CH}_{2}$, $5.71(1 \mathrm{H}, \mathrm{dt}, J 16.5,10.4,2-H) ; \delta_{\mathrm{C}}(100 \mathrm{MHz}) 0.1\left(\mathrm{CH}_{3}, \mathrm{Si}\left(\mathrm{CH}_{3}\right)_{3}\right), 5.2\left(\mathrm{CH}_{2}, \mathrm{OSi}\left(\mathrm{CH}_{2} \mathrm{CH}_{3}\right)\right), 5.4\left(\mathrm{CH}_{2}\right.$, $\left.\mathrm{OSi}\left(\mathrm{CH}_{2} \mathrm{CH}_{3}\right)\right), 6.3\left(\mathrm{CH}_{3}, \mathrm{OSi}\left(\mathrm{CH}_{2} \mathrm{CH}_{3}\right)\right), 6.5\left(\mathrm{CH}_{3}, \mathrm{OSi}\left(\mathrm{CH}_{2} \mathrm{CH}_{3}\right)\right), 14.1\left(\mathrm{CH}_{3}, \mathrm{C}-10\right), 22.6\left(\mathrm{CH}_{2}\right), 28.2$ $\left(\mathrm{CH}_{2}\right), 37.3\left(\mathrm{CH}_{2}\right), 40.1(\mathrm{CH}, \mathrm{C}-3), 40.4\left(\mathrm{CH}_{2}\right), 69.8(\mathrm{CH}, \mathrm{CH}(\mathrm{O})), 70.4(\mathrm{CH}, \mathrm{CH}(\mathrm{O})), 113.5\left(\mathrm{CH}_{2}, \mathrm{C}-1\right)$, $137.5(\mathrm{CH}, \mathrm{C}-2) ; \mathrm{m} / \mathrm{z}$ (TOF ES+) $351.3\left([\mathrm{M}+\mathrm{Na}]^{+}, 100 \% \text { ); HRMS m/z (TOF ES+) } 351.2151 \text { ([M+Na] }\right]^{+}$. $\mathrm{C}_{17} \mathrm{H}_{36} \mathrm{NaO}_{2} \mathrm{Si}_{2}$ requires 351.2152). 
$\left(3 R^{*}, 4 R^{*}, 6 R^{\star}\right)$ oxasilinane $8 f$<smiles>C=C[C@H]1[C@@H](OC)CC(CCCBr)O[Si]1(CC)CC</smiles>

\author{
$\mathrm{C}_{17} \mathrm{H}_{36} \mathrm{O}_{2} \mathrm{Si}_{2}$ \\ Exact Mass: 328.22538 \\ Mol. Wt.: 328.63754 \\ C, $62.13 ; \mathrm{H}, 11.04 ; \mathrm{O}, 9.74 ; \mathrm{Si}, 17.09 \%$
}

HPLC: $t_{\mathrm{R}}=69.0 \mathrm{~min} ; v_{\max }(\mathrm{film}) / \mathrm{cm}^{-1} 2957 \mathrm{~s}, 1626 \mathrm{w}(\mathrm{C}=\mathrm{C}), 1456 \mathrm{~m}, 1252 \mathrm{~m}, 1057 \mathrm{~m}, 963 \mathrm{w}, 897 \mathrm{w}$, $841 \mathrm{~m}, 725 \mathrm{w} ; \delta_{\mathrm{H}}(400 \mathrm{MHz}) 0.10\left(9 \mathrm{H}, \mathrm{s}, \mathrm{Si}\left(\mathrm{CH}_{3}\right)_{3}\right), 0.48-0.84\left(4 \mathrm{H}\right.$, stack, OSi $\left.\left(\mathrm{CH}_{2} \mathrm{CH}_{3}\right)_{2}\right), 0.89(3 \mathrm{H}, \mathrm{t}, J$ 7.0, 10- $\left.\mathrm{CH}_{3}\right), 0.95\left(3 \mathrm{H}, \mathrm{t}, J 7.8, \mathrm{OSi}\left(\mathrm{CH}_{2} \mathrm{CH}_{3}\right)\right), 0.96\left(3 \mathrm{H}, \mathrm{t}, J\right.$ 7.8, OSi $\left.\left(\mathrm{CH}_{2} \mathrm{CH}_{3}\right)\right), 1.23-1.41(6 \mathrm{H}$, stack, 9- $\left.\mathrm{CH}_{2}, 8-\mathrm{CH}_{2}, 7-\mathrm{CH}_{\mathrm{a}} \mathrm{H}_{\mathrm{b}}, 5-\mathrm{H}_{\text {pseudoax. }}\right), 1.43-1.49\left(1 \mathrm{H}, \mathrm{m}, 7-\mathrm{CH}_{\mathrm{a}} \mathrm{H}_{b}\right), 1.65(1 \mathrm{H}$, ddd, J 13.9, 4.9, 1.7, 5$\left.H_{\text {pseudoeq. }}\right), 1.83(1 \mathrm{H}, \mathrm{dd}, J$ 10.2, 2.8, 3-H), 4.08-4.16 $(1 \mathrm{H}, \mathrm{m}, 6-H), 4.19-4.23(1 \mathrm{H}, \mathrm{m}, 4-H), 4.84(1 \mathrm{H}, \mathrm{dd}$, $J$ 10.2, 2.2, 1- $\left.H_{c i s}\right), 4.88\left(1 \mathrm{H}, \mathrm{dd}, J 17.1,2.2,1-H_{\text {trans }}\right), 5.88(1 \mathrm{H}, \mathrm{dt}, J 17.1,10.2,2-H) ; \delta_{\mathrm{C}}(100 \mathrm{MHz}) 0.2$ $\left(\mathrm{CH}_{3}, \mathrm{Si}\left(\mathrm{CH}_{3}\right)_{3}\right), 6.6\left(2 \times \mathrm{CH}_{3}, 2 \times \mathrm{CH}_{2}, \mathrm{OSi}\left(\mathrm{CH}_{2} \mathrm{CH}_{3}\right)_{2}\right), 14.1\left(\mathrm{CH}_{3}, \mathrm{C}-10\right), 22.7\left(\mathrm{CH}_{2}\right), 27.6\left(\mathrm{CH}_{2}\right), 38.0$ $\left(\mathrm{CH}_{2}\right), 38.8(\mathrm{CH}, \mathrm{C}-3), 42.3\left(\mathrm{CH}_{2}\right), 67.0(\mathrm{CH}, \mathrm{CH}(\mathrm{O})), 71.6(\mathrm{CH}, \mathrm{CH}(\mathrm{O})), 112.5\left(\mathrm{CH}_{2}, \mathrm{C}-1\right), 138.3(\mathrm{CH}$, $\mathrm{C}-2) ; \mathrm{m} / \mathrm{z}$ (TOF ES+) $351.3\left([\mathrm{M}+\mathrm{Na}]^{+}, 100 \%\right) ; \mathrm{HRMS} \mathrm{m} / \mathrm{z}$ (TOF ES+) 351.2151 ([M+Na] $]^{+}$. $\mathrm{C}_{17} \mathrm{H}_{36} \mathrm{NaO}_{2} \mathrm{Si}_{2}$ requires 351.2152).

$\left(3 S^{*}, 4 R^{*}, 6 R^{\star}\right)$ Dec-1-ene-3,4,6-triol $9 f$<smiles>C=CC(O)C(O)CC(O)CCCC</smiles>

\author{
$\mathrm{C}_{10} \mathrm{H}_{20} \mathrm{O}_{3}$ \\ Exact Mass: 188.14125 \\ Mol. Wt.: 188.26400 \\ C, $63.80 ; \mathrm{H}, 10.71 ; \mathrm{O}, 25.50 \%$
}

$\mathrm{H}_{2} \mathrm{O}_{2}\left(1.207 \mathrm{~g}, 60 \%\right.$ in $\left.\mathrm{H}_{2} \mathrm{O}, 21.30 \mathrm{mmol}\right), \mathrm{KHCO}_{3}(320 \mathrm{mg}, 3.20 \mathrm{mmol})$ and $\mathrm{KF}$ (309 mg, $\left.5.32 \mathrm{mmol}\right)$ were added to a solution of the products from the allylation of aldehyde $6 \mathrm{f}(663 \mathrm{mg}, 0.72 \mathrm{mmol}$ of oxasilinane 7f) in MeOH:THF $(1: 1,11 \mathrm{~mL})$ and the resulting mixture was stirred for 5 days. Aqueous work-up and purification by flash column chromatography ( $70 \rightarrow 90 \%$ EtOAc in hexane) afforded triol 9f as a colorless viscous oil (89 mg, 65\%); $R_{f}=0.30$ (70\% EtOAc in hexane); $v_{\max }\left(\right.$ film) $/ \mathrm{cm}^{-1} 3368 \mathrm{br} \mathrm{s}$ 
$(\mathrm{OH}), 2956 \mathrm{~s}, 2930 \mathrm{~s}, 2861 \mathrm{~s}, 1645 \mathrm{w}(\mathrm{C}=\mathrm{C}), 1431 \mathrm{~s}, 1379 \mathrm{~m}, 1318 \mathrm{~m}, 1189 \mathrm{~m}, 1126 \mathrm{~s}, 1054 \mathrm{~s}, 995 \mathrm{~s}, 925 \mathrm{~s}$, $847 \mathrm{~m}, 731 \mathrm{~m} ; \delta_{\mathrm{H}}(300 \mathrm{MHz}) 0.86\left(3 \mathrm{H}, \mathrm{t}, J 6.8, \mathrm{CH}_{3}\right), 1.20-1.65\left(8 \mathrm{H}\right.$, stack, $\left.4 \times \mathrm{CH}_{2}\right), 3.15-3.92(4 \mathrm{H}$, stack including [3.77-3.84 (1H, m, CHOH), $3.88(1 \mathrm{H}, \mathrm{dt}, J$ 9.6, 3.1, $\mathrm{CHOH})], 2 \times \mathrm{OH}, 2 \times \mathrm{CHOH}), 4.07-$ $4.22(2 \mathrm{H}$, stack including [4.10 (1H, app. t, J 5.9, CHOH)], $\mathrm{CHOH}, \mathrm{OH}), 5.23\left(1 \mathrm{H}, \mathrm{d}, J 10.3,=\mathrm{CH}_{c i s}\right)$, $5.31\left(1 \mathrm{H}, J 17.3,=\mathrm{CHH}_{\text {trans }}\right), 5.86\left(1 \mathrm{H}, \mathrm{ddd}, 17.3,10.3,5.9, \mathrm{CH}=\mathrm{CH}_{2}\right) ; \delta_{\mathrm{C}}(75 \mathrm{MHz}) 13.6\left(\mathrm{CH}_{3}, \mathrm{C}-10\right)$, $22.3\left(\mathrm{CH}_{2}\right), 27.2\left(\mathrm{CH}_{2}\right), 36.7\left(\mathrm{CH}_{2}\right), 37.6\left(\mathrm{CH}_{2}\right), 72.3(\mathrm{CH}, \mathrm{CHOH}), 74.9(\mathrm{CH}, \mathrm{CHOH}), 75.7(\mathrm{CH}$, $\mathrm{CHOH}), 117.5\left(\mathrm{CH}_{2},=\mathrm{CH}_{2}\right), 136.6\left(\mathrm{CH}, \mathrm{CH}=\mathrm{CH}_{2}\right) ; \mathrm{m} / \mathrm{z}(\mathrm{Cl}+) 206\left[\left(\mathrm{M}+\mathrm{NH}_{4}\right)^{+}, 100 \%\right], 188(\mathrm{M})^{+}(26), 148$ (10), 172 (8); $\mathrm{HRMS} \mathrm{m} / \mathrm{z}(\mathrm{Cl}+)$ 206.175561 ([M+NH$]_{4}^{+} . \mathrm{C}_{10} \mathrm{H}_{24} \mathrm{NO}_{3}$ requires 206.175619).

Acetonide protection of triol 9f: 1-(2,2-dimethyl-5-vinyl-[1,3]dioxolan-4-yl)-hexan-2-ol 11f and 1(6-butyl-2,2-dimethyl-[1,3]dioxan-4-yl)-prop-2-en-1-ol 12f
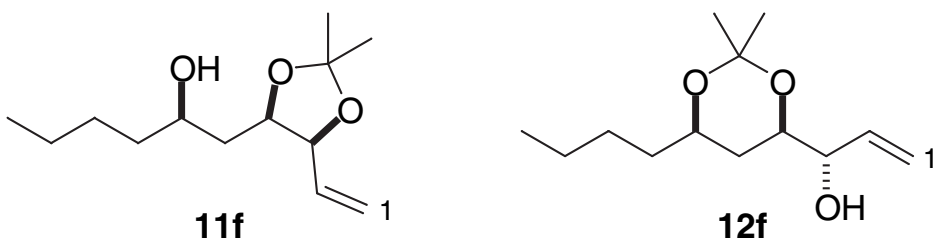

$$
\mathrm{C}_{13} \mathrm{H}_{24} \mathrm{O}_{3}
$$

Exact Mass: 228.17255

Mol. Wt.: 228.32786

C, 68.38; H, 10.59; O, $21.02 \%$

$\mathrm{Na}_{2} \mathrm{SO}_{4}(40 \mathrm{mg})$ and $p \mathrm{TsOH} \cdot \mathrm{H}_{2} \mathrm{O}(3 \mathrm{mg}, 16 \mu \mathrm{mol})$ were added to a solution of trio/ $9 \mathrm{f}(30 \mathrm{mg}, 0.16$ $\mathrm{mmol})$ in acetone $(1.6 \mathrm{~mL})$ and the resulting mixture was stirred overnight. Aqueous work-up afforded alcohols $11 \mathrm{f}$ and $12 \mathrm{f}$ as a colorless oil and an inseparable mixture (1:1 by ${ }^{1} \mathrm{H}$ NMR) (35 mg, quantitative); Data reported on the mixture of $11 \mathrm{f}$ and $12 \mathrm{f}: \delta_{\mathrm{H}}\left(\mathrm{C}_{6} \mathrm{D}_{6}, 300 \mathrm{MHz}\right) 0.82(3 \mathrm{H}, \mathrm{t}, J 7.0,1 \times$ $\left.\mathrm{CH}_{3}\left(\mathrm{CH}_{2}\right)_{3}\right), 0.92\left(3 \mathrm{H}, \mathrm{t}, \mathrm{J} 7.4,1 \times \mathrm{CH}_{3}\left(\mathrm{CH}_{2}\right)_{3}\right), 1.12-1.65\left(28 \mathrm{H}\right.$, stack including [1.22 $\left(3 \mathrm{H}, \mathrm{s}, 1 \times \mathrm{CCH}_{3}\right)$, $\left.1.27\left(3 \mathrm{H}, \mathrm{s}, 1 \times \mathrm{CCH}_{3}\right), 1.40\left(3 \mathrm{H}, \mathrm{s}, 1 \times \mathrm{CCH}_{3}\right), 1.46\left(3 \mathrm{H}, \mathrm{s}, 1 \times \mathrm{CCH}_{3}\right)\right], 8 \times \mathrm{CH}_{2}, 2 \times \mathrm{C}_{\left.\left(\mathrm{CH}_{3}\right)_{2}\right), 2.25-}$ $2.42(1 \mathrm{H}, \mathrm{br} \mathrm{s}, 1 \times \mathrm{OH}), 2.96(1 \mathrm{H}, \mathrm{br} \mathrm{s}, 1 \times \mathrm{OH}), 3.49-3.60(1 \mathrm{H}, \mathrm{m}, 1 \times n \mathrm{BuCH}), 3.66(1 \mathrm{H}, \mathrm{ddd}, J 11.8$, 4.0, 3.0, $\left.1 \times \mathrm{CH}(\mathrm{O}) \mathrm{CH}(\mathrm{O}) \mathrm{CH}=\mathrm{CH}_{2}\right), 3.69-3.79(1 \mathrm{H}, \mathrm{m}, 1 \times n \mathrm{BuCH}), 4.06(1 \mathrm{H}, \mathrm{ddd}, J 10.0,6.2,2.9,1 \times$ $\left.\mathrm{CH}(\mathrm{O}) \mathrm{CH}(\mathrm{O}) \mathrm{CH}=\mathrm{CH}_{2}\right), 4.16\left(1 \mathrm{H}\right.$, app. t, J 5.0, $\left.1 \times \mathrm{CH}(\mathrm{O}) \mathrm{CH}=\mathrm{CH}_{2}\right), 4.30(1 \mathrm{H}$, app. t, J 6.9, $1 \times$ $\left.\mathrm{CH}(\mathrm{O}) \mathrm{CH}=\mathrm{CH}_{2}\right), 5.01\left(1 \mathrm{H}, \mathrm{d}, J 10.3,1 \times 1-\mathrm{H}_{\text {cis }}\right), 5.14\left(1 \mathrm{H}\right.$, app. dt, J 10.3, 1.8, $\left.1 \times 1-H_{c i s}\right), 5.15(1 \mathrm{H}, \mathrm{d}$, $\left.J 17.3,1 \times 1-H_{\text {trans }}\right), 5.43\left(1 \mathrm{H}\right.$, app. dt, J 17.3, 1.8, $\left.1 \times 1-H_{\text {trans }}\right), 5.65(1 \mathrm{H}, \mathrm{ddd}, J 17.3,10.3,6.9,1 \times 2-$ $H), 5.85(1 \mathrm{H}$, ddd, J 17.3, 10.3, 5.0, $1 \times 2-H) ; \delta_{\mathrm{C}}\left(\mathrm{C}_{6} \mathrm{D}_{6}, 75 \mathrm{MHz}\right) 13.6\left(\mathrm{CH}_{3}, 1 \times \mathrm{CH}_{3}\left(\mathrm{CH}_{2}\right)_{3}\right), 13.7\left(\mathrm{CH}_{3}\right.$, $\left.1 \times \mathrm{CH}_{3}\left(\mathrm{CH}_{2}\right)_{3}\right), 19.3\left(\mathrm{CH}_{3}, 1 \times \mathrm{CCH}_{3} 12 \mathrm{f}\right), 22.5\left(\mathrm{CH}_{2}\right), 22.6\left(\mathrm{CH}_{2}\right), 25.1\left(\mathrm{CH}_{3}, 1 \times \mathrm{CCH}_{3} 11 \mathrm{f}\right), 27.0$ $\left(\mathrm{CH}_{2}\right), 27.5\left(\mathrm{CH}_{2}\right), 27.6\left(\mathrm{CH}_{3}, 1 \times \mathrm{CCH}_{3}\right.$ 11f), $29.8\left(\mathrm{CH}_{3}, 1 \times \mathrm{CCH}_{3} 12 \mathrm{f}\right), 30.2\left(\mathrm{CH}_{2}\right), 36.2\left(\mathrm{CH}_{2}\right), 37.3$ $\left(\mathrm{CH}_{2}\right), 37.5\left(\mathrm{CH}_{2}\right), 68.5(\mathrm{CH}, 1 \times \mathrm{CH}(\mathrm{O})), 70.9(\mathrm{CH}, 1 \times \mathrm{CH}(\mathrm{O})), 72.1(\mathrm{CH}, 1 \times \mathrm{CH}(\mathrm{O})), 74.1(\mathrm{CH}, 1 \times$ $\mathrm{CH}(\mathrm{O})), 78.4(\mathrm{CH}, 1 \times \mathrm{CH}(\mathrm{O})), 79.7(\mathrm{CH}, 1 \times \mathrm{CH}(\mathrm{O})), 98.5$ (quat. $\mathrm{C}, \mathrm{C}\left(\mathrm{CH}_{3}\right)_{2}$ 12f), 108.7 (quat. $\mathrm{C}$, $\mathrm{C}\left(\mathrm{CH}_{3}\right)_{2}$ 11f), $115.8\left(\mathrm{CH}_{2}, 1 \times \mathrm{C}-1\right), 117.5\left(\mathrm{CH}_{2}, 1 \times \mathrm{C}-1\right), 135.0(\mathrm{CH}, 1 \times \mathrm{C}-2), 137.1(\mathrm{CH}, 1 \times \mathrm{C}-2)$. 


\title{
Allylation reaction of Aldehyde $6 \mathrm{~g}$ :
}

TMSOTf $(217 \mu \mathrm{L}, 1.20 \mathrm{mmol})$ was added to a solution of aldehyde $\mathbf{6 g}(394 \mathrm{mg}, 1.20 \mathrm{mmol})$ and 2,6DTBMP (296 mg, $1.40 \mathrm{mmol})$ in $\mathrm{CH}_{2} \mathrm{Cl}_{2}(12 \mathrm{~mL})$ at $-78{ }^{\circ} \mathrm{C}$ and the reaction mixture was stirred for $8 \mathrm{~h}$. Aqueous work-up and removal of the solvent afforded compounds $\mathbf{7 g}$ and $\mathbf{8} \mathbf{g}$ (40:15; inseparable mixture) as a yellow liquid ( $687 \mathrm{mg}, 55 \%$, remaining material being diene products). This was used in the following step without any further purification. Analytically pure samples of compounds $\mathbf{7 g}$ and $\mathbf{8 g}$ were obtained as colorless oils by preparative HPLC ( $t=0 \rightarrow t=40$ min, $50 \rightarrow 100 \% \mathrm{MeCN}_{\text {in }} \mathrm{H}_{2} \mathrm{O}$ ); $7 \mathbf{g} t_{\mathrm{R}}=66.9 \mathrm{~min}$ and $8 \mathbf{g} t_{\mathrm{R}}=76.0 \mathrm{~min}$.

\section{$\left(3 S^{\star}, 4 R^{\star}, 6 R^{\star}\right)$ oxasilinane $7 \mathrm{~g}$}<smiles>CC[Si]1(CC)OC(CC(C)C)C[C@H](OC)C1C=[IH]</smiles>$$
\mathrm{C}_{17} \mathrm{H}_{36} \mathrm{O}_{2} \mathrm{Si}_{2}
$$ \\ Exact Mass: 328.22538 \\ Mol. Wt.: 328.63754 \\ C, $62.13 ; \mathrm{H}, 11.04 ; \mathrm{O}, 9.74 ; \mathrm{Si}, 17.09 \%$
}

HPLC: $t_{\mathrm{R}}=66.9 \mathrm{~min} ; v_{\max }($ film $) / \mathrm{cm}^{-1} 3077 \mathrm{w}, 2956 \mathrm{~s}, 2877 \mathrm{~s}, 1628 \mathrm{~m}(\mathrm{C}=\mathrm{C}), 1468 \mathrm{~m}, 1415 \mathrm{w}, 1375 \mathrm{~m}$, $1347 \mathrm{w}, 1330 \mathrm{w}, 1304 \mathrm{w}, 1252 \mathrm{~s}, 1154 \mathrm{~m}, 1116 \mathrm{~m}, 1073 \mathrm{~s}, 990 \mathrm{~m}, 959 \mathrm{~m}, 935 \mathrm{~m}, 893 \mathrm{~m}, 867 \mathrm{~m}, 841 \mathrm{~s}, 747 \mathrm{~m}$, $724 \mathrm{~m}, 656 \mathrm{w}, 631 \mathrm{~m} ; \delta_{\mathrm{H}}(400 \mathrm{MHz}) 0.09\left(9 \mathrm{H}, \mathrm{s}, \mathrm{Si}\left(\mathrm{CH}_{3}\right)_{3}\right), 0.56\left(2 \mathrm{H}, \mathrm{q}, J \mathrm{J.6}, \mathrm{OSi}\left(\mathrm{CH}_{2} \mathrm{CH}_{3}\right)\right), 0.67-0.78$ $\left(2 \mathrm{H}, \mathrm{m}, \mathrm{OSi}\left(\mathrm{CH}_{2} \mathrm{CH}_{3}\right)\right), 0.89\left(6 \mathrm{H}, \mathrm{d}, J 6.6, \mathrm{CH}\left(\mathrm{CH}_{3}\right)_{2}\right), 0.91\left(3 \mathrm{H}, \mathrm{t}, J 7.8, \mathrm{OSi}\left(\mathrm{CH}_{2} \mathrm{CH}_{3}\right)\right), 0.97(3 \mathrm{H}, \mathrm{t}, J$ 7.8, OSi $\left(\mathrm{CH}_{2} \mathrm{CH}_{3}\right)$ ), $1.15\left(1 \mathrm{H}\right.$, ddd, J 13.4, 8.3, 4.9, 7- $\left.\mathrm{CH}_{a} \mathrm{H}_{\mathrm{b}}\right), 1.50\left(1 \mathrm{H}\right.$, ddd, J 13.4, 8.8, 5.6, 7- $\left.\mathrm{CH}_{\mathrm{a}} H_{b}\right)$, $1.61\left(1 \mathrm{H}, \mathrm{ddd}, J 13.9,7.1,3.7,5-\mathrm{CH}_{a} \mathrm{H}_{\mathrm{b}}\right), 1.68\left(1 \mathrm{H}\right.$, ddd, $\left.J 13.9,6.9,2.8,5-\mathrm{CH}_{\mathrm{a}} \mathrm{H}_{\mathrm{b}}\right), 1.73-1.83(1 \mathrm{H}, \mathrm{m}$, 8-H), $1.89(1 \mathrm{H}, \mathrm{dd}, J 10.4,7.0,3-H), 4.07(1 \mathrm{H}, \mathrm{td}, J$ 7.0, 2.7, 4-H), 4.16-4.22 $(1 \mathrm{H}, \mathrm{m}, 6-H), 4.86-4.95$ $\left(2 \mathrm{H}\right.$, stack, 1-CH $\mathrm{CH}_{2}, 5.72(1 \mathrm{H}, \mathrm{dt}, \mathrm{J} 16.3,10.5,2-\mathrm{H}) ; \delta_{\mathrm{C}}(100 \mathrm{MHz}) 0.1\left(\mathrm{CH}_{3}, \mathrm{Si}\left(\mathrm{CH}_{3}\right)_{3}\right), 5.30\left(\mathrm{CH}_{2}\right.$, $\left.\mathrm{OSi}\left(\mathrm{CH}_{2} \mathrm{CH}_{3}\right)\right), 5.34\left(\mathrm{CH}_{2}\right.$, OSi $\left.\left(\mathrm{CH}_{2} \mathrm{CH}_{3}\right)\right), 6.2\left(\mathrm{CH}_{3}, \mathrm{OSi}\left(\mathrm{CH}_{2} \mathrm{CH}_{3}\right)\right), 6.5\left(\mathrm{CH}_{3}, \mathrm{OSi}\left(\mathrm{CH}_{2} \mathrm{CH}_{3}\right)\right), 22.1$ $\left(\mathrm{CH}_{3}, \mathrm{C}-9\right), 23.1\left(\mathrm{CH}_{3}, \mathrm{C}-9\right)$ ') $24.6(\mathrm{CH}, \mathrm{C}-8), 39.9(\mathrm{CH}, \mathrm{C}-3), 40.8\left(\mathrm{CH}_{2}, \mathrm{C}-5\right), 46.8\left(\mathrm{CH}_{2}, \mathrm{C}-7\right), 67.5$ (CH, C-6), $70.6(\mathrm{CH}, \mathrm{C}-4), 113.5\left(\mathrm{CH}_{2}, \mathrm{C}-1\right), 137.5(\mathrm{CH}, \mathrm{C}-2) ; \mathrm{m} / \mathrm{z}$ (TOF ES+) $351.1\left([\mathrm{M}+\mathrm{Na}]^{+}, 100 \%\right.$ ); HRMS $m / z$ (TOF ES+) $351.2146\left([\mathrm{M}+\mathrm{Na}]^{+} . \mathrm{C}_{17} \mathrm{H}_{36} \mathrm{NaO}_{2} \mathrm{Si}_{2}\right.$ requires 351.2152). 
<smiles>C=C[C@@H]1[C@@H](OC)CC(CC(C)C)O[Si]1(CC)CC</smiles>

$$
\mathrm{C}_{17} \mathrm{H}_{36} \mathrm{O}_{2} \mathrm{Si}_{2}
$$

Exact Mass: 328.22538

Mol. Wt.: 328.63754

C, $62.13 ; \mathrm{H}, 11.04 ; \mathrm{O}, 9.74 ; \mathrm{Si}, 17.09 \%$

HPLC: $t_{R}=76.0 \mathrm{~min} ; v_{\max }($ film $) / \mathrm{cm}^{-1} 3076 \mathrm{w}, 2957 \mathrm{~s}, 2877 \mathrm{~m}, 1627 \mathrm{w}(\mathrm{C}=\mathrm{C}), 1462 \mathrm{~m}, 1416 \mathrm{w}, 1370 \mathrm{w}$, $1324 \mathrm{w}, 1252 \mathrm{~s}, 1170 \mathrm{w}, 1126 \mathrm{~m}, 1084 \mathrm{~s}, 1058 \mathrm{~s}, 1004 \mathrm{~m}, 968 \mathrm{~m}, 938 \mathrm{w}, 898 \mathrm{~m}, 868 \mathrm{~m}, 841 \mathrm{~s}, 808 \mathrm{w}, 757 \mathrm{~m}$, $723 \mathrm{~m}, 658 \mathrm{w} ; \delta_{\mathrm{H}}(500 \mathrm{MHz}) 0.09\left(9 \mathrm{H}, \mathrm{s}, \mathrm{Si}\left(\mathrm{CH}_{3}\right)_{3}\right), 0.47-0.86\left(4 \mathrm{H}\right.$, stack, $\left.\mathrm{OSi}\left(\mathrm{CH}_{2} \mathrm{CH}_{3}\right)_{2}\right), 0.800(3 \mathrm{H}, \mathrm{d}, \mathrm{J}$ 6.5, 9- $\left.\mathrm{CH}_{3}\right), 0.805\left(3 \mathrm{H}, \mathrm{d}, J 6.5,9\right.$ '- $\left.\mathrm{CH}_{3}\right), 0.93\left(6 \mathrm{H}, \mathrm{t}, J\right.$ 7.9, OSi $\left.\left(\mathrm{CH}_{2} \mathrm{CH}_{3}\right)_{2}\right), 1.10(1 \mathrm{H}$, ddd, J 13.5, 8.3, 4.8, 7- $\left.\mathrm{CH}_{a} \mathrm{H}_{\mathrm{b}}\right), 1.35\left(1 \mathrm{H}, \mathrm{dd}, J\right.$ 13.8, 11.1, 5- $\left.\mathrm{CH}_{\text {pseudoax. }}\right), 1.43\left(1 \mathrm{H}, \mathrm{ddd}, J 13.5,8.2,5.7,7-\mathrm{CH}_{\mathrm{a}} \mathrm{H}_{\mathrm{b}}\right), 1.62$ $\left(1 \mathrm{H}\right.$, ddd, J 13.8, 5.0, 1.8, 5- $\left.\mathrm{CH}_{\text {pseudoeq. }}\right), 1.73-1.82(1 \mathrm{H}, \mathrm{m}, 8-\mathrm{H}), 1.82(1 \mathrm{H}, \mathrm{dd}, J 10.1,2.7,3-H), 4.16-$ $4.25(2 \mathrm{H}$, stack, 4- $\mathrm{H}, 6-H), 4.80-4.92\left(2 \mathrm{H}\right.$, stack, 1- $\left.\mathrm{CH}_{2}\right), 5.86(1 \mathrm{H}, \mathrm{dt}, J 17.3,10.2,2-H) ; \delta_{\mathrm{C}}(125 \mathrm{MHz})$ $0.2\left(\mathrm{CH}_{3}, \mathrm{Si}\left(\mathrm{CH}_{3}\right)_{3}\right), 4.8\left(\mathrm{CH}_{2}, \mathrm{Si}\left(\mathrm{CH}_{2} \mathrm{CH}_{3}\right)\right), 5.4\left(\mathrm{CH}_{2}, \mathrm{OSi}\left(\mathrm{CH}_{2} \mathrm{CH}_{3}\right)\right), 6.6\left(2 \times \mathrm{CH}_{3}, \mathrm{OSi}\left(\mathrm{CH}_{2} \mathrm{CH}_{3}\right)_{2}\right)$, $\left.22.2\left(\mathrm{CH}_{3}, \mathrm{C}-9\right), 23.2\left(\mathrm{CH}_{3}, \mathrm{C}^{\prime}\right)^{\prime}\right), 24.4(\mathrm{CH}, \mathrm{C}-8), 38.9(\mathrm{CH}, \mathrm{C}-3), 42.9\left(\mathrm{CH}_{2}, \mathrm{C}-5\right), 47.5\left(\mathrm{CH}_{2}, \mathrm{C}-7\right)$, $65.0(\mathrm{CH}), 71.6(\mathrm{CH}), 112.4\left(\mathrm{CH}_{2}, \mathrm{C}-1\right), 138.3(\mathrm{CH}, \mathrm{C}-2) ; \mathrm{m} / \mathrm{z}(\mathrm{TOF} \mathrm{ES}+) 351.3\left([\mathrm{M}+\mathrm{Na}]^{+}, 100 \%\right)$; HRMS $m / z$ (TOF ES+) $351.2154\left([\mathrm{M}+\mathrm{Na}]^{+} . \mathrm{C}_{17} \mathrm{H}_{36} \mathrm{NaO}_{2} \mathrm{Si}_{2}\right.$ requires 351.2152).

\title{
$\left(3 S^{*}, 4 R^{\star}, 6 R^{\star}\right)$ 8-Methyl-non-1-ene-3,4,6-triol 9g
}<smiles>CC(C)CC(O)CC(O)C(O)C=[IH]</smiles>

\author{
$\mathrm{C}_{10} \mathrm{H}_{20} \mathrm{O}_{3}$ \\ Exact Mass: 188.14125 \\ Mol. Wt.: 188.26400 \\ C, $63.80 ; \mathrm{H}, 10.71 ; \mathrm{O}, 25.50 \%$
}

$\mathrm{H}_{2} \mathrm{O}_{2}\left(1.247 \mathrm{~g}, 60 \%\right.$ in $\left.\mathrm{H}_{2} \mathrm{O}, 22.00 \mathrm{mmol}\right), \mathrm{KHCO}_{3}(330 \mathrm{mg}, 3.30 \mathrm{mmol})$ and $\mathrm{KF}(320 \mathrm{mg}, 5.50 \mathrm{mmol})$ were added to a solution of the products from the allylation of aldehyde $6 \mathrm{~g}(684 \mathrm{mg}, 0.77 \mathrm{mmol}$ of oxasilinane $\mathbf{7 g})$ in $\mathrm{MeOH}$ :THF $(1: 1,11 \mathrm{~mL})$ and the resulting mixture was stirred for 5 days. Aqueous work-up and purification by flash column chromatography (70 $\rightarrow 90 \%$ EtOAc in hexane) afforded triol $9 \mathrm{~g}$ as a colorless viscous oil $(87 \mathrm{mg}, 60 \%) ; \mathrm{R}_{\mathrm{f}}=0.29$ (70\% EtOAc in hexane); $v_{\max }\left(\right.$ film) $/ \mathrm{cm}^{-1} 3315 \mathrm{br} \mathrm{s}$ 
$(\mathrm{OH}), 2954 \mathrm{~s}, 1645 \mathrm{~m}(\mathrm{C}=\mathrm{C}), 1469 \mathrm{~s}, 1434 \mathrm{~s}, 1368 \mathrm{~s}, 1311 \mathrm{~s}, 1192 \mathrm{~m}, 1171 \mathrm{~m}, 1147 \mathrm{~s}, 1077 \mathrm{~s}, 995 \mathrm{~s}, 924 \mathrm{~s}$, $881 \mathrm{~m}, 852 \mathrm{~m}, 813 \mathrm{~m}, 738 \mathrm{~s} ; \delta_{\mathrm{H}}(300 \mathrm{MHz}) 0.89\left(6 \mathrm{H}, \mathrm{d}, J 6.6,\left(\mathrm{CH}_{3}\right)_{2} \mathrm{CH}\right), 1.13-1.28\left(1 \mathrm{H}, \mathrm{m}, 7-\mathrm{H}_{\mathrm{a}} \mathrm{H}_{\mathrm{b}}\right)$, 1.36-1.63 $\left(3 \mathrm{H}\right.$, stack, $\left.7-\mathrm{H}_{\mathrm{a}} \mathrm{H}_{\mathrm{b}}, 5-\mathrm{H}\right), 1.66-1.80\left(1 \mathrm{H}, \mathrm{m},\left(\mathrm{CH}_{3}\right)_{2} \mathrm{CH}\right), 3.22(1 \mathrm{H}$, br s, $1 \times \mathrm{OH}), 3.32(1 \mathrm{H}, \mathrm{br}$ $\mathrm{s}, 1 \times \mathrm{OH}), 3.85-4.20(4 \mathrm{H}$, stack including [3.86-3.95 $(2 \mathrm{H}$, stack, $2 \times \mathrm{CHOH}), 4.08-4.15(1 \mathrm{H}, \mathrm{m}, 1 \times$ $\mathrm{CHOH})], 1 \times \mathrm{OH}, 3 \times \mathrm{CHOH}), 5.24\left(1 \mathrm{H}, \mathrm{d}, J 10.7,=\mathrm{CH}_{\text {cis }} \mathrm{H}_{\text {trans }}\right), 5.31\left(1 \mathrm{H}, \mathrm{d}, J 17.3,=\mathrm{CH}_{\text {cis }} H_{\text {trans }}\right), 5.86$ $\left(1 \mathrm{H}\right.$, ddd, $J$ 17.3, 10.7, 6.1, $\left.\mathrm{CH}=\mathrm{CH}_{2}\right) ; \delta_{\mathrm{C}}(75 \mathrm{MHz}) 21.8\left(\mathrm{CH}_{3}, 1 \times\left(\mathrm{CH}_{3}\right)_{2} \mathrm{CH}\right), 22.9\left(\mathrm{CH}_{3}, 1 \times\left(\mathrm{CH}_{3}\right)_{2} \mathrm{CH}\right)$, $23.9\left(\mathrm{CH},\left(\mathrm{CH}_{3}\right)_{2} \mathrm{CH}\right), 37.2\left(\mathrm{CH}_{2}\right), 47.1\left(\mathrm{CH}_{2}\right), 70.4(\mathrm{CH}, 1 \times \mathrm{CHOH}), 74.9(\mathrm{CH}, 1 \times \mathrm{CHOH}), 75.7(\mathrm{CH}$, $1 \times \mathrm{CHOH}), 117.5\left(\mathrm{CH}_{2},=\mathrm{CH}_{2}\right), 136.6(\mathrm{CH},=\mathrm{CH}) ; \mathrm{m} / \mathrm{z}(\mathrm{Cl}+) 206\left[\left(\mathrm{M}+\mathrm{NH}_{4}\right)^{+}, 100 \%\right], 188(\mathrm{M})^{+}(19), 153$ (6); HRMS $m / z(C l+) 206.175418\left(\left[\mathrm{M}+\mathrm{NH}_{4}\right]^{+} . \mathrm{C}_{10} \mathrm{H}_{24} \mathrm{NO}_{3}\right.$ requires 206.175619).

Acetonide protection of triol 9g: 1-(2,2-dimethyl-5-vinyl-[1,3]dioxolan-4-yl)-4-methyl-pentan-2-ol $11 \mathrm{~g}$ and 1-(6-isobutyl-2,2-dimethyl-[1,3]dioxan-4-yl)-prop-2-en-1-ol 12g
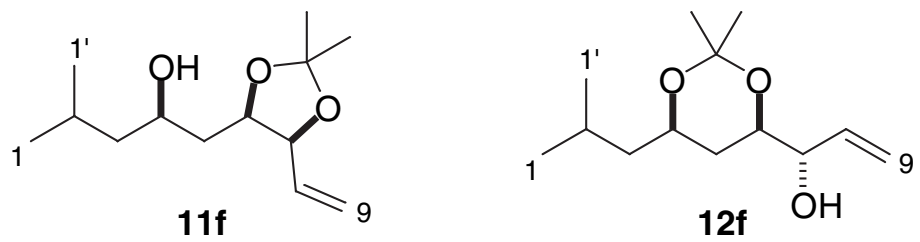

$$
\mathrm{C}_{13} \mathrm{H}_{24} \mathrm{O}_{3}
$$

Exact Mass: 228.17255

Mol. Wt.: 228.32786

C, $68.38 ; \mathrm{H}, 10.59 ; \mathrm{O}, 21.02 \%$

$\mathrm{Na}_{2} \mathrm{SO}_{4}(40 \mathrm{mg})$ and $p \mathrm{TsOH} \cdot \mathrm{H}_{2} \mathrm{O}(3 \mathrm{mg}, 16 \mu \mathrm{mol})$ were added to a solution of trio/ $9 \mathrm{~g}(32 \mathrm{mg}, 0.17$ $\mathrm{mmol})$ in acetone $(1.7 \mathrm{~mL})$ and the resulting mixture was stirred overnight. Aqueous work-up afforded acetonides $11 \mathrm{~g}$ and $\mathbf{1 2} \mathrm{g}$ as a colorless oil and an inseparable mixture (53:47 by ${ }^{1} \mathrm{H}$ NMR) (36 $\mathrm{mg}$, quantitative); Data reported on the mixture of $11 \mathrm{~g}$ and $\mathbf{1 2 g}$ : $\delta_{\mathrm{H}}\left(\mathrm{C}_{6} \mathrm{D}_{6}, 300 \mathrm{MHz}\right) 0.86(3 \mathrm{H}, \mathrm{d}, J 2.9,1 \times$ $\left.\left(\mathrm{CH}_{3}\right)_{2} \mathrm{CH}\right), 0.89\left(3 \mathrm{H}, \mathrm{d}, J 2.9,1 \times\left(\mathrm{CH}_{3}\right)_{2} \mathrm{CH}\right), 1.06\left(3 \mathrm{H}, \mathrm{d}, J\right.$ 7.0, $\left.1 \times\left(\mathrm{CH}_{3}\right)_{2} \mathrm{CH}\right), 1.09(3 \mathrm{H}, \mathrm{d}, J 6.6,1 \times$ $\left.\left(\mathrm{CH}_{3}\right)_{2} \mathrm{CH}\right), 1.09-1.73\left(2 \mathrm{H}\right.$, stack including $\left[1.31\left(3 \mathrm{H}, \mathrm{s}, 1 \times\left(\mathrm{CH}_{3}\right)_{\text {pseudoax. }}\right), 1.35(3 \mathrm{H}, \mathrm{s}, 1 \times\right.$ $\left.\left.\left(\mathrm{CH}_{3}\right)_{\text {pseudoax. }}\right), 1.50\left(3 \mathrm{H}, \mathrm{s}, 1 \times\left(\mathrm{CH}_{3}\right)_{\text {pseudoeq. }}\right), 1.54\left(3 \mathrm{H}, \mathrm{s}, 1 \times\left(\mathrm{CH}_{3}\right)_{\text {pseudoeq. }}\right)\right], 2 \times 3-\mathrm{H}_{2}, 2 \times 5-\mathrm{H}_{2}, 2 \times$ $\left.\mathrm{CH}\left(\mathrm{CH}_{3}\right)_{2}\right), 1.90-2.02(1 \mathrm{H}, \mathrm{m}, 1 \times 2-\mathrm{H}), 2.03-2.18(1 \mathrm{H}, \mathrm{m}, 1 \times 2-\mathrm{H}), 2.18(1 \mathrm{H}, \mathrm{br} \mathrm{s}, 1 \times \mathrm{OH}), 3.08(1 \mathrm{H}$, br s, $1 \times \mathrm{OH}), 3.61-3.73(2 \mathrm{H}$, stack, $1 \times 4-H, 1 \times 6-H), 3.85(1 \mathrm{H}$, tdd, J 12.1, 3.7, 2.6, $1 \times 4-H), 4.03$ (1H, ddd, J 10.7, 7.0, 2.9, $1 \times 6-H), 4.15(1 \mathrm{H}$, app. t, J 4.4, $1 \times 7-H), 4.29(1 \mathrm{H}$, app. t, J 6.6, $1 \times 7-H)$, $5.00\left(1 \mathrm{H}, \mathrm{d}, J 10.3,1 \times 9-H_{\text {cis }}\right), 5.14\left(1 \mathrm{H}, \mathrm{d}, J 10.3,1 \times 9-H_{\text {cis }}\right), 5.15\left(1 \mathrm{H}, \mathrm{d}, J 17.6,1 \times 9-H_{\text {trans }}\right), 5.43$ $\left(1 \mathrm{H}, \mathrm{td}, J 17.3,1.8,1 \times 9-H_{\text {trans }}\right), 5.63(1 \mathrm{H}, \mathrm{ddd}, J 17.6,10.3,5.1,7.0,1 \times 8-H), 5.83(1 \mathrm{H}, \mathrm{ddd}, J 17.3$, 10.3, 5.1, $1 \times 8-H) ; \delta_{\mathrm{C}}\left(\mathrm{C}_{6} \mathrm{D}_{6}, 75 \mathrm{MHz}\right) 19.2\left(\mathrm{CH}_{3},\left(\mathrm{CH}_{3}\right)_{\text {pseudoax. }} 12 \mathrm{~g}\right),[21.7,22.9,23.2,24.1(\mathrm{CH}$ and $\left.\mathrm{CH}_{3},\left(\mathrm{CH}_{3}\right)_{2} \mathrm{CH} 11 \mathrm{~g},\left(\mathrm{CH}_{3}\right)_{2} \mathrm{CH} \mathbf{1 2} \mathbf{g}\right)$, some overlap], $25.0\left(\mathrm{CH}_{3},\left(\mathrm{CH}_{3}\right)_{\text {pseudoax. }} 11 \mathrm{~g}\right), 27.6\left(\mathrm{CH}_{3}\right.$, $\left(\mathrm{CH}_{3}\right)_{\text {pseudoeq. }}$ 11g), $29.8\left(\mathrm{CH}_{3},\left(\mathrm{CH}_{3}\right)_{\text {pseudoeq. }} 12 \mathrm{~g}\right), 30.6\left(\mathrm{CH}_{2}\right), 38.1\left(\mathrm{CH}_{2}\right), 45.5\left(\mathrm{CH}_{2}\right), 47.0\left(\mathrm{CH}_{2}\right), 66.4$ 
$(\mathrm{CH}), 69.0(\mathrm{CH}), 72.1(\mathrm{CH}), 74.1(\mathrm{CH}), 78.4(\mathrm{CH}), 79.7(\mathrm{CH}), 98.4$ (quat. $\mathrm{C},\left(\mathrm{CH}_{3}\right)_{2} \mathrm{C} \mathrm{12g}$ ), 108.7 (quat. C, $\left.\left(\mathrm{CH}_{3}\right)_{2} \mathrm{C} 11 \mathrm{~g}\right), 115.8\left(\mathrm{CH}_{2}, \mathrm{C}-9\right), 117.5\left(\mathrm{CH}_{2}, \mathrm{C}-9\right), 135.0(\mathrm{CH}, \mathrm{C}-8), 137.0(\mathrm{CH}, \mathrm{C}-8)$.

\title{
Allylation reaction of Aldehyde 6h:
}

TMSOTf ( $145 \mu \mathrm{L}, 0.80 \mathrm{mmol}$ ) was added to a solution of aldehyde $6 \mathrm{~h}(325 \mathrm{mg}, 0.80 \mathrm{mmol})$ and 2,6DTBMP (203 mg, $0.96 \mathrm{mmol})$ in $\mathrm{CH}_{2} \mathrm{Cl}_{2}(8 \mathrm{~mL})$ at $-78{ }^{\circ} \mathrm{C}$ and the reaction mixture was stirred for $8 \mathrm{~h}$. Aqueous work-up and removal of the solvent afforded compounds $7 \mathbf{h}$ and $\mathbf{8} \mathbf{h}$ (40:15; inseparable mixture) as a yellow liquid (532 mg, $55 \%$, remaining material being diene products). This was used in the following step without any further purification. Analytically pure samples of compounds $\mathbf{7 h}$ and $\mathbf{8 h}$ were obtained as colorless oils by preparative HPLC ( $t=0 \rightarrow t=40 \mathrm{~min}, 50 \rightarrow 100 \% \mathrm{MeCN}_{\text {in }} \mathrm{H}_{2} \mathrm{O}$ ); $7 \mathrm{~h} t_{\mathrm{R}}=38.6 \mathrm{~min}$ and $8 \mathrm{~h} t_{\mathrm{R}}=48.7 \mathrm{~min}$.

$\left(3 S^{*}, 4 R^{*}, 6 R^{\star}\right)$ oxasilinane $7 \mathrm{~h}$<smiles>C=CC1[C@@H](OC)CC(CCO[C]c2ccccc2)O[Si]1(CC)CC</smiles>

\author{
$\mathrm{C}_{22} \mathrm{H}_{38} \mathrm{O}_{3} \mathrm{Si}_{2}$ \\ Exact Mass: 406.23595 \\ Mol. Wt.: 406.70632 \\ C, $64.97 ; \mathrm{H}, 9.42 ; \mathrm{O}, 11.80 ; \mathrm{Si}, 13.81 \%$
}

HPLC: $t_{R}=38.6 \mathrm{~min} ; v_{\max }(\mathrm{film}) / \mathrm{cm}^{-1} 3074 \mathrm{w}, 3031 \mathrm{w}, 2954 \mathrm{~s}, 2915 \mathrm{~m}, 2876 \mathrm{~m}, 1805 \mathrm{w}, 1627 \mathrm{w}(\mathrm{C}=\mathrm{C})$, $1496 \mathrm{w}, 1455 \mathrm{~m}, 1414 \mathrm{w}, 1363 \mathrm{w}, 1251 \mathrm{~s}, 1204 \mathrm{w}, 1152 \mathrm{~m}, 1114 \mathrm{~s}, 1075 \mathrm{~s}, 1028 \mathrm{~m}, 996 \mathrm{~m}, 933 \mathrm{~m}, 873 \mathrm{~m}$, 840s, 745m, 733m, 697m; $\delta_{\mathrm{H}}(500 \mathrm{MHz}) 0.10\left(9 \mathrm{H}, \mathrm{s}, \mathrm{Si}\left(\mathrm{CH}_{3}\right)_{3}\right), 0.56\left(2 \mathrm{H}, \mathrm{q}, J \mathrm{~J} .9, \mathrm{OSi}\left(\mathrm{CH}_{2} \mathrm{CH}_{3}\right)\right), 0.66-$ $0.80\left(2 \mathrm{H}, \mathrm{m}, \mathrm{OSi}\left(\mathrm{CH}_{2} \mathrm{CH}_{3}\right)\right), 0.91\left(3 \mathrm{H}, \mathrm{t}, J 7.9, \mathrm{OSi}\left(\mathrm{CH}_{2} \mathrm{CH}_{3}\right)\right), 0.98\left(3 \mathrm{H}, \mathrm{t}, J 7.9, \mathrm{OSi}\left(\mathrm{CH}_{2} \mathrm{CH}_{3}\right)\right), 1.65$ $\left(1 \mathrm{H}\right.$, ddd, J 13.8, 7.0, 3.5, 5- $\left.\mathrm{CH}_{a} \mathrm{H}_{\mathrm{b}}\right), 1.71-1.86\left(3 \mathrm{H}\right.$, stack, 5- $\left.\mathrm{CH}_{\mathrm{a}} \mathrm{H}_{b}, 7-\mathrm{CH}_{2}\right), 1.88(1 \mathrm{H}, \mathrm{dd}, J$ 10.3, 6.7, 3-H), 3.59-3.64 (2H, m, 8-CH $\mathrm{CH}_{2}, 4.08(1 \mathrm{H}$, app. td, J 6.8, 2.2, 4-H), 4.27-4.33 (1H, m, 6-H), $4.50(1 \mathrm{H}, \mathrm{A}$ of $\mathrm{AB}, J$ 12.1, $\left.\mathrm{PhCH}_{A} \mathrm{H}_{\mathrm{B}}\right), 4.52\left(1 \mathrm{H}, \mathrm{B}\right.$ of $\mathrm{AB}, J$ 12.1, $\left.\mathrm{PhCH}_{\mathrm{A}} H_{B}\right), 4.92\left(1 \mathrm{H}, \mathrm{d}, J 11.3,1-H_{\text {cis }}\right), 4.93(1 \mathrm{H}$, $\left.\mathrm{d}, J 16.0,1-H_{\text {trans }}\right), 5.68-5.77(1 \mathrm{H}, \mathrm{m}, 2-H), 7.26-7.36(5 \mathrm{H}$, stack, $\mathrm{PhH}) ; \delta_{\mathrm{C}}(125 \mathrm{MHz}) 0.12\left(\mathrm{CH}_{3}\right.$, $\left.\mathrm{Si}\left(\mathrm{CH}_{3}\right)_{3}\right), 5.3\left(\mathrm{CH}_{2}, \mathrm{OSi}\left(\mathrm{CH}_{2} \mathrm{CH}_{3}\right)\right), 5.4\left(\mathrm{CH}_{2}, \mathrm{OSi}\left(\mathrm{CH}_{2} \mathrm{CH}_{3}\right)\right), 6.3\left(\mathrm{CH}_{3}, \mathrm{OSi}\left(\mathrm{CH}_{2} \mathrm{CH}_{3}\right)\right), 6.5\left(\mathrm{CH}_{3}\right.$, OSi $\left.\left(\mathrm{CH}_{2} \mathrm{CH}_{3}\right)\right), 37.9\left(\mathrm{CH}_{2}, \mathrm{C}-7\right), 39.8(\mathrm{CH}, \mathrm{C}-3), 40.6\left(\mathrm{CH}_{2}, \mathrm{C}-5\right), 66.7(\mathrm{CH}, \mathrm{C}-6), 67.4\left(\mathrm{CH}_{2}, \mathrm{C}-8\right), 70.5$ $(\mathrm{CH}, \mathrm{C}-4), 73.1\left(\mathrm{CH}_{2}, \mathrm{PhCH}_{2}\right), 113.7\left(\mathrm{CH}_{2}, \mathrm{C}-1\right), 127.5(\mathrm{CH}, \mathrm{Ph}), 127.6(\mathrm{CH}, \mathrm{Ph}), 128.3(\mathrm{CH}, \mathrm{Ph})$, 137.4 (CH, C-2), 138.6 (quat. C, ipsoPh); m/z (TOF ES+) 429.1 ([M+Na] ${ }^{+}, 100 \%$ ); HRMS m/z (TOF $\mathrm{ES}+) 429.2277\left([\mathrm{M}+\mathrm{Na}]^{+} . \mathrm{C}_{22} \mathrm{H}_{38} \mathrm{NaO}_{3} \mathrm{Si}_{2}\right.$ requires 429.2257). 


\section{$\left(3 R^{\star}, 4 R^{\star}, 6 R^{\star}\right)$ oxasilinane $8 \mathrm{~h}$}<smiles>C=C[C@H]1[C@@H](OC)CC(CCO[C]c2ccccc2)O[Si]1(CC)CC</smiles>

$$
\mathrm{C}_{22} \mathrm{H}_{38} \mathrm{O}_{3} \mathrm{Si}_{2}
$$

Exact Mass: 406.23595

Mol. Wt.: 406.70632

C, 64.97; H, 9.42; O, 11.80; Si, 13.81\%

HPLC: $t_{\mathrm{R}}=48.7 \mathrm{~min} ; v_{\max }($ film $) / \mathrm{cm}^{-1} 3073 \mathrm{w}, 3031 \mathrm{w}, 3044 \mathrm{w}, 2955 \mathrm{~s}, 2914 \mathrm{~m}, 2875 \mathrm{~s}, 1626 \mathrm{w}(\mathrm{C}=\mathrm{C})$, 1416w, 1363w, 1316w, 1251s, 1204w, 1170w, 1112m, 1080s, 1054s, 1028m, 1003m, 962m, 897w, $879 \mathrm{w}, 860 \mathrm{~m}, 841 \mathrm{~s}, 807 \mathrm{w}, 731 \mathrm{~m}, 696 \mathrm{~m} ; \delta_{\mathrm{H}}(400 \mathrm{MHz}) 0.10\left(9 \mathrm{H}, \mathrm{s}, \mathrm{Si}\left(\mathrm{CH}_{3}\right)_{3}\right), 0.45-0.59(2 \mathrm{H}, \mathrm{m}$, $\left.\mathrm{OSi}\left(\mathrm{CH}_{2} \mathrm{CH}_{3}\right)\right), 0.53-0.90\left(2 \mathrm{H}, \mathrm{m}, \mathrm{OSi}\left(\mathrm{CH}_{2} \mathrm{CH}_{3}\right)\right), 0.95\left(3 \mathrm{H}, \mathrm{t}, J 7.8, \mathrm{OSi}\left(\mathrm{CH}_{2} \mathrm{CH}_{3}\right)\right), 0.96(3 \mathrm{H}, \mathrm{t}, J 7.9$, $\left.\mathrm{OSi}\left(\mathrm{CH}_{2} \mathrm{CH}_{3}\right)\right), 1.44\left(1 \mathrm{H}\right.$, ddd, J 14.0, 11.2, 1.2, 5- Hseudoax. $\left._{\text {. }}\right) 1.66(1 \mathrm{H}$, ddd, J 14.0, 4.9, 2.0, 5$\left.H_{\text {pseudoeq. }}\right), 1.71-1.77\left(2 \mathrm{H}\right.$, stack, 7- $\left.\mathrm{CH}_{2}\right), 1.84(1 \mathrm{H}, \mathrm{dd}, \mathrm{J} 10.0,2.9,3-\mathrm{H}), 3.63\left(2 \mathrm{H}, \mathrm{t}, \mathrm{J} 6.7,8-\mathrm{CH}_{2}\right), 4.19-$ $4.25(1 \mathrm{H}, \mathrm{m}, 4-H), 4.30-4.38(1 \mathrm{H}, \mathrm{m}, 6-H), 4.50\left(2 \mathrm{H}, \mathrm{s}, \mathrm{PhCH}_{2}\right), 4.85\left(1 \mathrm{H}, \mathrm{dd}, J 10.2,2.2,1-\mathrm{H}_{\text {cis }}\right), 4.89$ $\left(1 \mathrm{H}, \mathrm{dd}, J 17.3,1.5,1-H_{\text {trans }}\right), 5.88\left(1 \mathrm{H}\right.$, app. dt, J 17.3, 10.1, 2-H), 7.26-7.36 (5H, stack, PhH); $\delta_{\mathrm{C}}(100$ $\mathrm{MHz}) 0.12\left(\mathrm{CH}_{3}, \mathrm{Si}\left(\mathrm{CH}_{3}\right)_{3}\right), 4.8\left(\mathrm{CH}_{2}, \mathrm{OSi}\left(\mathrm{CH}_{2} \mathrm{CH}_{3}\right)\right), 5.4\left(\mathrm{CH}_{2}, \mathrm{OSi}\left(\mathrm{CH}_{2} \mathrm{CH}_{3}\right)\right), 6.6\left(2 \times \mathrm{CH}_{3}\right.$, OSi $\left.\left(\mathrm{CH}_{2} \mathrm{CH}_{3}\right)_{2}\right), 38.3\left(\mathrm{CH}_{2}, \mathrm{C}-7\right), 38.7(\mathrm{CH}, \mathrm{C}-3), 42.7\left(\mathrm{CH}_{2}, \mathrm{C}-5\right), 64.4(\mathrm{CH}, \mathrm{C}-6), 67.3\left(\mathrm{CH}_{2}, \mathrm{C}-8\right)$, $71.5(\mathrm{CH}, \mathrm{C}-4), 73.0\left(\mathrm{CH}_{2}, \mathrm{PhCH}_{2}\right), 112.5\left(\mathrm{CH}_{2}, \mathrm{C}-1\right), 127.4(\mathrm{CH}, \mathrm{Ph}), 127.5(\mathrm{CH}, \mathrm{Ph}), 128.3(\mathrm{CH}, \mathrm{Ph})$, 138.2 (CH, C-2), 138.8 (quat. C, ipsoPh); $\mathrm{m} / \mathrm{z}$ (TOF ES+) 429.2 ([M+Na] $]^{+}, 100 \%$ ); HRMS m/z (TOF $\mathrm{ES}+) 429.2273\left([\mathrm{M}+\mathrm{Na}]^{+} . \mathrm{C}_{22} \mathrm{H}_{38} \mathrm{NaO}_{3} \mathrm{Si}_{2}\right.$ requires 429.2257).

$\left(3 S^{\star}, 4 R^{\star}, 6 R^{\star}\right)$ 8-Benzyloxy-oct-1-ene-3,4,6-triol 9h<smiles>OC(C=[IH])CC(O)CCOCc1ccccc1</smiles>

$\mathrm{C}_{15} \mathrm{H}_{22} \mathrm{O}_{4}$

Exact Mass: 266.15181

Mol. Wt.: 266.33278

C, $67.64 ; \mathrm{H}, 8.33 ; \mathrm{O}, 24.03 \%$ 
$\mathrm{H}_{2} \mathrm{O}_{2}$ (623 mg, 60\% in $\mathrm{H}_{2} \mathrm{O}, 11.0 \mathrm{mmol}$ ), $\mathrm{KHCO}_{3}$ (165 mg, $1.65 \mathrm{mmol}$ ) and $\mathrm{KF}$ (160 mg, $2.75 \mathrm{mmol}$ ) were added to a solution of the products from the allylation of aldehyde $6 \mathrm{~h}(356 \mathrm{mg}, 0.37 \mathrm{mmol}$ of oxasilinane $\mathbf{7 h})$ in MeOH:THF (1:1, $8 \mathrm{~mL})$ and the resulting mixture was stirred for 5 days. Aqueous work-up and purification by flash column chromatography ( $70 \rightarrow 90 \%$ EtOAc in hexane) afforded triol $9 \mathrm{~h}$ as a colorless viscous oil (92 $\mathrm{mg}, 63 \%) ; \mathrm{R}_{\mathrm{f}}=0.28\left(80 \%\right.$ EtOAc in hexane); $v_{\max }(\mathrm{film}) / \mathrm{cm}^{-1} 3392 \mathrm{~s} \mathrm{br}$ $(\mathrm{OH}), 3088 \mathrm{~m}, 3031 \mathrm{~m}, 2919 \mathrm{~s}, 2866 \mathrm{~s}, 1874 \mathrm{w}, 1737 \mathrm{w}, 1644 \mathrm{w}(\mathrm{C}=\mathrm{C}), 1496 \mathrm{~m}, 1454 \mathrm{~s}, 1428 \mathrm{~s}, 1365 \mathrm{~m}$, $1312 \mathrm{~m}, 1207 \mathrm{~m}, 1094 \mathrm{~s}, 1028 \mathrm{~s}, 996 \mathrm{~s}, 927 \mathrm{~m}, 849 \mathrm{w}, 799 \mathrm{~s}, 737 \mathrm{~s} ; \delta_{\mathrm{H}}(300 \mathrm{MHz}) 1.50-1.90(4 \mathrm{H}$, stack, $2 \times$ $\left.\mathrm{CH}_{2}\right)$, 3.59-3.78 (2H, stack), 3.83-3.95 $(1 \mathrm{H}, \mathrm{m}, 1 \times \mathrm{CHOH}), 4.04-4.18\left(2 \mathrm{H}\right.$, stack), $4.52\left(2 \mathrm{H}, \mathrm{s}, \mathrm{PhCH}_{2}\right)$, $5.22\left(1 \mathrm{H}, \mathrm{d}, \mathrm{J} 10.7,=\mathrm{CH}_{\text {cis }} \mathrm{H}_{\text {trans }}\right), 5.32\left(1 \mathrm{H}, \mathrm{d}, J 17.3,=\mathrm{CH}_{\text {cis }} H_{\text {trans }}\right), 5.85(1 \mathrm{H}, \mathrm{ddd}, J 17.3,10.7,5.9$, $\left.\mathrm{CH}=\mathrm{CH}_{2}\right), 7.25-7.42(5 \mathrm{H}$, stack, $\mathrm{PhH})$, the resonance for the $3 \times \mathrm{OHs} v$ br and not resolved; $\delta_{\mathrm{C}}(75$ $\mathrm{MHz}) 36.8\left(\mathrm{CH}_{2}\right), 36.9\left(\mathrm{CH}_{2}\right), 68.9\left(\mathrm{CH}_{2}\right), 71.9(\mathrm{CH}, 1 \times \mathrm{CHOH}), 73.4\left(\mathrm{CH}_{2}\right), 74.5(\mathrm{CH}, 1 \times \mathrm{CHOH})$, $75.4(\mathrm{CH}, 1 \times \mathrm{CHOH}), 116.7\left(\mathrm{CH}_{2},=\mathrm{CH}_{2}\right), 127.7(\mathrm{CH}, \mathrm{Ph}), 127.8(\mathrm{CH}, \mathrm{Ph}), 128.5(\mathrm{CH}, \mathrm{Ph}), 136.5(\mathrm{CH}$, $\mathrm{CH}=\mathrm{CH}_{2}$ ), 137.6 (quat. C, ipsoPh); $\mathrm{m} / \mathrm{z}$ (TOF ES+) 289.1 ([M+Na] $]^{+}, 100 \%$ ); HRMS m/z (TOF ES + ) $289.1\left([\mathrm{M}+\mathrm{Na}]^{+}\right.$. $\mathrm{C}_{15} \mathrm{H}_{22} \mathrm{NaO}_{4}$ requires 289.1416).

Acetonide protection of triol 9h: 4-benzyloxy-1-(2,2-dimethyl-5-vinyl-[1,3]dioxolan-4-yl)-butan-2ol $11 \mathrm{~h}$ and 1-[6-(2-benzyloxy-ethyl)-2,2-dimethyl-[1,3]dioxan-4-yl]-prop-2-en-1-ol 12h<smiles>CC1(C)OC(C=[IH])C(CC(O)CCOCc2ccccc2)O1</smiles>

$11 \mathrm{~h}$<smiles>CC1(C)OC(CCOCc2ccccc2)CC([C@H](O)C=[IH])O1</smiles>

$12 \mathrm{~h}$

\author{
$\mathrm{C}_{18} \mathrm{H}_{26} \mathrm{O}_{4}$ \\ Exact Mass: 306.18311 \\ Mol. Wt.: 306.39664 \\ C, $70.56 ; \mathrm{H}, 8.55 ; \mathrm{O}, 20.89 \%$
}

$\mathrm{Na}_{2} \mathrm{SO}_{4}(40 \mathrm{mg})$ and $\mathrm{pTsOH} \cdot \mathrm{H}_{2} \mathrm{O}(3 \mathrm{mg}, 16 \mu \mathrm{mol})$ were added to a solution of triol $9 \mathrm{~h}$ (30 mg, 0.11 $\mathrm{mmol})$ in acetone $(1.6 \mathrm{~mL})$ and the resulting mixture was stirred overnight. Aqueous work-up afforded acetonides $11 \mathrm{~h}$ and $12 \mathrm{~h}$ as a colorless oil and as an inseparable mixture (56:44 by ${ }^{1} \mathrm{H}$ NMR) (33 mg, quantitative); Data reported on the mixture of $11 \mathrm{~h}$ and $12 \mathrm{~h}: \delta_{\mathrm{H}}\left(\mathrm{C}_{6} \mathrm{D}_{6}, 300 \mathrm{MHz}\right) 1.20-1.53(16 \mathrm{H}$, stack including $\left[1.25\left(3 \mathrm{H}, \mathrm{s},\left(\mathrm{CH}_{3}\right)_{\text {pseudoax. }} 11 \mathrm{~h}\right), 1.30\left(3 \mathrm{H}, \mathrm{s},\left(\mathrm{CH}_{3}\right)_{\text {pseudoax. }} \mathbf{1 2 h}\right), 1.46\left(3 \mathrm{H}, \mathrm{s},\left(\mathrm{CH}_{3}\right)_{\text {pseudoeq. }}\right.\right.$ 11h), $\left.\left.1.48\left(3 \mathrm{H}, \mathrm{s},\left(\mathrm{CH}_{3}\right)_{\text {pseudoeq. }} 12 \mathrm{~h}\right)\right], \mathrm{C}\left(\mathrm{CH}_{3}\right)_{2} 11 \mathrm{~h}, \mathrm{C}\left(\mathrm{CH}_{3}\right)_{2} 12 \mathrm{~h}, 1 \times \mathrm{CH}_{2} 11 \mathrm{~h}, 1 \times \mathrm{CH}_{2} 12 \mathrm{~h}\right), 1.67-$ $1.93\left(4 \mathrm{H}\right.$, stack, $\mathrm{BnOCH}_{2} \mathrm{CH}_{2} 11 \mathrm{~h}, \mathrm{BnOCH}_{2} \mathrm{CH}_{2}$ 12h), [(3.37-3.49), (3.50-3.61), (3.61-3.73), (3.904.01), (4.02-4.21), (4.28-4.41) 14H, $6 \times$ stack / m, $4 \times \mathrm{CH}_{2} \mathrm{O}, 6 \times \mathrm{CHO}, 5.04\left(1 \mathrm{H}, \mathrm{d}, \mathrm{J} 10.3,1-\mathrm{H}_{\text {cis }} 11 \mathrm{~h}\right)$, $5.16\left(1 \mathrm{H}\right.$, app. dt, J 10.3, 1.7, 1- $\left.H_{c i s} 12 \mathrm{~h}\right), 5.18\left(1 \mathrm{H}, \mathrm{d}, J 15.8,1-H_{\text {trans }} 11 \mathrm{~h}\right), 5.45(1 \mathrm{H}$, app. dt, J 17.3, 
$1.71-H_{\text {trans }}$ 12h), $5.69(1 \mathrm{H}$, ddd, J 15.8, 10.3, 7.0, 2-H 11h), $5.83(1 \mathrm{H}, \mathrm{ddd}, J 17.3,10.3,5.1,2-H 12 \mathrm{~h})$, 7.09-7.37 (10H, stack, $\mathrm{PhH} 11 \mathrm{~h}, \mathrm{PhH} 12 \mathrm{~h})$, resonances for $\mathrm{OH}$ not observed; $\delta_{\mathrm{C}}\left(\mathrm{C}_{6} \mathrm{D}_{6}, 75 \mathrm{MHz}\right) 20.0$ $\left(\mathrm{CH}_{3},\left(\mathrm{CH}_{3}\right)_{\text {pseudoax. }} 12 \mathrm{~h}\right), 25.7\left(\mathrm{CH}_{3},\left(\mathrm{CH}_{3}\right)_{\text {pseudoax. }} 11 \mathrm{~h}\right), 28.3\left(\mathrm{CH}_{3},\left(\mathrm{CH}_{3}\right)_{\text {pseudoeq. }} \mathbf{1 1} \mathbf{h}\right), 30.4\left(\mathrm{CH}_{3}\right.$, $\left(\mathrm{CH}_{3}\right)_{\text {pseudoeq. }}$ 12h), $30.7\left(\mathrm{CH}_{2}\right), 37.2\left(\mathrm{CH}_{2}\right), 37.8\left(\mathrm{CH}_{2}\right), 38.2\left(\mathrm{CH}_{2}\right), 66.0(\mathrm{CH}), 66.4\left(\mathrm{CH}_{2}\right), 68.1\left(\mathrm{CH}_{2}\right)$, $69.2(\mathrm{CH}), 72.3(\mathrm{CH}), 73.1\left(\mathrm{CH}_{2}\right), 73.2\left(\mathrm{CH}_{2}\right), 74.4(\mathrm{CH}), 78.0(\mathrm{CH}), 80.0(\mathrm{CH}), 98.8$ (quat. C, $\mathrm{C}\left(\mathrm{CH}_{3}\right)_{2}$ 12h), 108.7 (quat. $\mathrm{C}, \mathrm{C}\left(\mathrm{CH}_{3}\right)_{2}$ 11h), $115.9\left(\mathrm{CH}_{2}, \mathrm{C}-1\right), 117.5\left(\mathrm{CH}_{2}, \mathrm{C}-1\right), 127.66(\mathrm{CH}, \mathrm{Ph}), 127.68(\mathrm{CH}$, $\mathrm{Ph}), 127.74(\mathrm{CH}, \mathrm{Ph}), 127.78(\mathrm{CH}, \mathrm{Ph}), 128.56(\mathrm{CH}, \mathrm{Ph}), 128.58(\mathrm{CH}, \mathrm{Ph}), 135.1(\mathrm{CH}, \mathrm{C}-2), 136.9$ (CH, C-2), 139.2(quat. C, ipsoPh), 139.4 (quat. C, ipsoPh). 